\title{
The BMP antagonist gremlin 1 contributes to the development of cortical excitatory neurons, motor balance and fear responses
}

\author{
Mari Ichinose $^{1,2}$, Nobumi Suzuki ${ }^{1,2}$, Tongtong Wang ${ }^{1,2}$, Hiroki Kobayashi $i^{1,2,3}$, Laura Vrbanac ${ }^{1,2}$, Jia Q. Ng $^{1,2}$, \\ Josephine A. Wright ${ }^{1,2}$, Tamsin R. M. Lannagan ${ }^{1,2}$, Krystyna A. Gieniec ${ }^{1,2}$, Martin Lewis ${ }^{4,5}$, Ryota Ando ${ }^{3}$, \\ Atsushi Enomoto ${ }^{3}$, Simon Koblar ${ }^{1,5}$, Paul Thomas ${ }^{1,2}$, Daniel L. Worthley ${ }^{2}$ and Susan L. Woods ${ }^{1,2, *}$
}

\begin{abstract}
Bone morphogenetic protein (BMP) signaling is required for early forebrain development and cortical formation. How the endogenous modulators of BMP signaling regulate the structural and functional maturation of the developing brain remains unclear. Here, we show that expression of the BMP antagonist Grem1 marks committed layer $\mathrm{V}$ and $\mathrm{VI}$ glutamatergic neurons in the embryonic mouse brain. Lineage tracing of Grem1-expressing cells in the embryonic brain was examined by administration of tamoxifen to pregnant Grem1creERT; Rosa26LSLTdtomato mice at 13.5 days post coitum (dpc), followed by collection of embryos later in gestation. In addition, at $14.5 \mathrm{dpc}$, bulk mRNA-seq analysis of differentially expressed transcripts between FACS-sorted Grem1-positive and -negative cells was performed. We also generated Emx1-cre-mediated Grem1 conditional knockout mice (Emx1-Cre;Grem1 $1^{\text {flox/flox })}$ in which the Grem1 gene was deleted specifically in the dorsal telencephalon. Grem1 $1^{\text {Emx } 1 c K O}$ animals had reduced cortical thickness, especially layers $\mathrm{V}$ and $\mathrm{VI}$, and impaired motor balance and fear sensitivity compared with littermate controls. This study has revealed new roles for Grem1 in the structural and functional maturation of the developing cortex.
\end{abstract}

KEY WORDS: Grem1, BMP, Cortical development, Excitatory neuron, Mouse

\section{INTRODUCTION}

During development of the nervous system, bone morphogenetic protein (BMP) signaling has important roles in the promotion of dorsal identity, and the regulation of cell proliferation and differentiation. Yet BMP function in the maturation of neurons in vivo is poorly understood. Misregulation of BMP signaling has been suggested to contribute to human neurodevelopmental conditions such as autism spectrum disorders (Yamasaki et al., 2015; Chen et al., 2017); however, a detailed understanding of the exact role for BMP signaling in these disorders is lacking, in line with our

${ }^{1}$ School of Medicine, Faculty of Health and Medical Sciences, University of Adelaide, SA 5000, Australia. ${ }^{2}$ Precision Medicine, South Australian Health and Medical Research Institute, Adelaide, SA 5000, Australia. ${ }^{3}$ Department of Pathology, Nagoya University Graduate School of Medicine, Nagoya 466-8560, Japan. ${ }^{4}$ Department of Psychiatry, College of Medicine and Public Health, Flinders University, Bedford Park, SA 5001, Australia. ${ }^{5}$ Lifelong Health, South Australian Health and Medical Research Institute, Adelaide, SA 5000, Australia.

*Author for correspondence (susan.woods@adelaide.edu.au)

(D) M.L., 0000-0002-3332-3776; S.L.W., 0000-0002-8955-2017

This is an Open Access article distributed under the terms of the Creative Commons Attribution License (http://creativecommons.org/licenses/by/4.0), which permits unrestricted use, distribution and reproduction in any medium provided that the original work is properly attributed.

Handling Editor: Paola Arlotta

Received 12 August 2020; Accepted 15 June 2021 imperfect knowledge of the regulation of the BMP pathway in normal brain development.

The BMP ligands (BMP2/4/5/6/7) bind to type I (BmprIA, BmprIB, Acvr1) and type II (BmprII, ActrIIA, and ActrIIB) receptors. BMP ligands are expressed broadly in the telencephalon during early gestation in mice and then become more tightly localized to the choroid plexus at 13.5 days post coitum (dpc) (Furuta et al., 1997). BMPs have also been derived from the mesenchyme of the brain, such as meninges and endothelial cells (Imura et al., 2008; Choe and Pleasure, 2018). Both type I and II BMP receptors are expressed in the telencephalon during development, but this is restricted in adulthood to BmprII in the cortex and hippocampus and ActrIIA/IIB in the dentate gyrus (Söderström et al., 1996). Aside from this spatiotemporal regulation of receptors, the BMP signaling pathway is also regulated by a family of secreted extracellular antagonists that directly bind to the BMP ligands to prevent interactions with BMP receptors both in development and disease (Ali and Brazil, 2014). Antagonists such as gremlin 1 (Grem1), noggin (Nog) and chordin (Chrd) have been shown to inhibit BMP action in a range of different cell types and developmental stagespecific contexts to provide exquisite spatiotemporal regulation of the pathway. The roles of Nog and Chrd have been partially elucidated: they are required for forebrain development (Bachiller et al., 2000), as well as to create a niche for adult hippocampal neurogenesis (Lim et al., 2000; Sun et al., 2007). The expression and function of Grem1 in the developing brain has not yet been determined. Grem1 is an extracellular secreted antagonist of BMP2/4/7 that signals to intestinal stem cells in the gut (Worthley et al., 2015) and plays a crucial role in Xenopus dorsalization (Hsu et al., 1998) and limb and kidney formation (Khokha et al., 2003). Likewise the role of Grem1 in the normal adult CNS is unmapped territory to date, outside of previous work in the pathogenic state of glioma (Yan et al., 2014; Guan et al., 2017; Fu et al., 2018).

BMP signaling has been implicated in the regulation of forebrain patterning during early embryogenesis, working together with other signaling pathways such as fibroblast growth factor (FGF), Wnt and Notch. High levels of BMP activity suppress anterior neural development, whereas abrogation of BMP signaling can promote neural specification (Wilson and Houart, 2004; Lamb et al., 1993; Bachiller et al., 2000). In vitro the addition of BMP2 and 4 to mouse neural stem cell cultures represses cell proliferation (Mathieu et al., 2008). BMP ligands regulate neuronal differentiation as well as determination of glial cell fate, by promoting astrocyte differentiation at the expense of oligodendrocytic fates (Mathieu et al., 2008; Yun et al., 2004; Sun and Xu, 2010; Gomes et al., 2003).

Radial glial cells (RGCs) begin to divide asymmetrically to start producing neurons at around $11.5 \mathrm{dpc}$ in mice. RGC daughter cells detach from the ventricle and form the first neuronal layer of the preplate by $13.5 \mathrm{dpc}$ (Haubensak et al., 2004). Subsequently born 
neurons migrate along RGCs and start to form the cortical plate, separating the preplate into Cajal-Retzius cells in the marginal zone and subplate neurons (Nichols and Olson, 2010; Marin-Padilla, 1978). The neocortex develops in an inside-out manner, with deep layers emerging first and superficial layers last. These neurons differentiate into glutamatergic pyramidal neurons, whereas inhibitory interneurons are born in the subcortical ganglionic eminences. Deep-layer pyramidal neurons (DLPNs, layer V and VI) have both intratelenchephalic projections to superficial cortical layers and extracortical projections to other brain regions (Saiki et al., 2017). Multiple molecular mechanisms regulate this corticogenesis (Kowalczyk et al., 2009; Franco et al., 2012; Chen et al., 2005); however, the role of BMP signaling in cortical layer formation and functional maturation of neurons has only been reported in limited studies so far. In utero electroporation of BMP7 to murine $14.5 \mathrm{dpc}$ cortical ventricular cells impaired neuronal migration, suggesting that BMP signaling regulates neuronal positioning and migration (Choe and Pleasure, 2018). BMPs also regulate dendritogenesis and neurite growth in vitro (Lee-Hoeflich et al., 2004; Matsuura et al., 2007). This is consistent with a recent study that suggests perturbation of BMP signaling by delivery of a dominant-negative version of BMP IB receptor affects migration, polarity and dendritogenesis of mouse cortical neurons in vivo (Saxena et al., 2018).

To further establish the role of BMP signaling in forebrain development and neuronal function, we focus here on the expression and function of the BMP antagonist Grem1 in the developing mouse brain. We first assessed Grem 1 expression using transgenic Grem 1 reporter mice in the dorsal telencephalon and developing neocortex. Next, to investigate the role of Grem1 in the developing brain, we used transcriptomic analyses of sorted mouse Grem1-expressing cells and single cell RNA-sequence (scRNAseq) data, combined with mouse neural stem/progenitor cell (NSPC) culture ex vivo. Lastly, to examine the functional contribution of Grem1 to cortical development, we conditionally deleted Grem1 in the dorsal telencephalon and undertook behavioral testing of mutant animals and littermate controls.

\section{RESULTS \\ Grem1-expressing cells are located in the dorsal telencephalon and give rise to deep-layer neocortical neurons}

Grem1 RNA was first detected by in situ hybridization (ISH) in the mouse dorsal telencephalon at $13.5 \mathrm{dpc}$ and expression was maintained through embryonic development until $20.5 \mathrm{dpc}$ (Fig. S1A). To further assess Greml expression in the embryonic mouse brain, we used transgenic Grem1creERT; Rosa26LSLTdtomato reporter mice, in which tamoxifen treatment results in expression of TdTomato in cells in which the Greml enhancer and promoter sequences are active, and the progeny of those cells (Worthley et al., 2015). Pregnant GremlcreERT; Rosa26LSLTdtomato mice were administered tamoxifen at $11.5 \mathrm{dpc}$, the time point at which RGCs begin dividing in the developing cortex, and embryonic brains were collected 24 h later. Consistent with our Grem1 ISH analysis, no TdTomato $^{+}$cells were observed, confirming that Greml is not yet expressed in the brain at this early time-point (Fig. S1B). Next, we administered tamoxifen to pregnant dams at $13.5 \mathrm{dpc}$ when Grem 1 is first expressed, and collected embryonic brains at 14.5, 17.5 and $20.5 \mathrm{dpc}$ (Fig. 1A,B). From this time point we observed cells expressing TdTomato $24 \mathrm{~h}$ after tamoxifen administration located in the lower cortical plate and subplate of the dorsal telencephalon, with dendrites extending to the pia mater (Fig. 1B,C). ISH confirmed that Grem1 RNA was detected in almost all TdTomato $^{+}$cells at $14.5 \mathrm{dpc}$, confirming that the reporter line recapitulates endogenous Grem1 expression (Fig. 1D,E). Six days later at $20.5 \mathrm{dpc}$, almost all layer V TdTomato ${ }^{+}$cells still expressed Grem1 RNA, whereas a significantly lower percentage of TdTomato $^{+}$layer VI neurons continued to express Greml RNA (Fig. 1D,E). Further co-staining of $\mathrm{TdTomato}^{+}$cells with cell typeand layer-specific markers showed that, at $20.5 \mathrm{dpc}$, the TdTomato ${ }^{+}$ cells have become Ctip2 ${ }^{+}\left(\mathrm{Bcll} 1 \mathrm{~b}^{+}\right)$layer V pyramidal neurons with dendrites extending to the pia mater and layer VI neurons (Fig. 1I; Fig. S1C). TdTomato ${ }^{+}$cells did not express the layer II-IV marker, CDP (Cux1) (Fig. S1C). Grem1 mRNA levels dramatically decreased after birth as determined by qRT-PCR analysis of total RNA isolated from mouse brain cortex at birth, postnatal day (P) 10 and 4 weeks post-birth (Fig. S1D).

To investigate when cells that become $\mathrm{Greml}^{+}$are born during mouse cortical development, we administered the thymidine analogue bromodeoxyuridine (BrdU) concomitantly with tamoxifen to pregnant Grem1creERT; Rosa26LSLTdtomato dams at $13.5 \mathrm{dpc}$ when it will be incorporated into dividing RGCs and intermediate progenitor cells (IPs) (Fig. 1A). We found that $55 \%$ of TdTomato $^{+}$layer $\mathrm{V}$ pyramidal neurons cells were labelled with BrdU at $17.5 \mathrm{dpc}$ (Fig. 1F,G), demonstrating that these cells are derived from progenitors at embryonic day (E)13.5, with Greml then expressed in committed layer V/VI neurons migrating over the following 3 days towards the cortical plate. Fewer TdTomato ${ }^{+}$cells $(17 \%)$ in layer VI were $\mathrm{BrdU}^{+}$(Fig. $\left.1 \mathrm{~F}, \mathrm{G}\right)$, consistent with layer VI neurons starting to be born before E13.5. At $14.5 \mathrm{dpc}$, TdTomato ${ }^{+}$ cells were also $\mathrm{Ki}^{-} 7^{-}\left(\mathrm{Mki67}^{-}\right)$, suggesting that they were nonproliferative (Fig. S1E). We subsequently assessed whether TdTomato $^{+}$cells express a marker of post-mitotic mature neurons, NeuN (Rbfox3), by immunohistochemistry (IHC). At $14.5 \mathrm{dpc}$ most TdTomato $^{+}$cells were $\mathrm{NeuN}^{-}$and were located in the subplate, and lower $\mathrm{NeuN}^{+}$cortical plate (Fig. 1H). In contrast, later in gestation at $20.5 \mathrm{dpc}$ almost all $\mathrm{TdTomato}^{+}$cells in Ctip2strongly-positive layer $\mathrm{V}$ neurons and approximately half of Ctip2weakly-positive layer VI neurons expressed NeuN (Fig. 1I,J). Together, these data suggest that Grem 1 starts being expressed by postmitotic cortical neurons destined for deep layers at E13.5 (Fig. 1C).

\section{Global transcriptomic analysis defines transcript modules enriched in Grem1-expressing cells}

To further characterize Grem 1 expressing cells in the developing mouse brain, we undertook mRNA-seq analysis on $\mathrm{TdTomato}^{+}$and TdTomato $^{-}$cells from the telencephalon. Pregnant Grem 1 creERT; Rosa26LSLTdtomato mice were administered tamoxifen at $13.5 \mathrm{dpc}$ and embryonic brains were collected at $14.5 \mathrm{dpc}$. At this time point almost all TdTomato $^{+}$cells expressed endogenous Grem1 RNA (Fig. 1D,E). Dissociated brain cells were flow sorted and live TdTomato $^{+}$and TdTomato $^{-}$cells were collected for transcriptomic analysis. TdTomato ${ }^{+}$cells accounted for $5.1 \pm 2.1 \%$ of all live cells ( $n=5$; mean \pm s.d.) (Fig. 2A). Bulk mRNA sequencing of TdTomato ${ }^{+}$ and TdTomato $^{-}$populations and analysis of 1845 differentially expressed genes (DEG) between the two cell populations revealed that Grem 1 was significantly upregulated in TdTomato $^{+}$cells and BMP transcriptional target genes (Id1/3/4) were significantly downregulated [adjusted $P$-value (FDR) $\leq 0.05$; Fig. $2 \mathrm{~B}$ ]. We next undertook a correlation analysis to identify modules of the 1845 DEGs that are coordinately regulated in TdTomato ${ }^{+}$cells (Fig. S2). Shared membership of a module can suggest genes that together perform a particular function. The clustering tree depicts the 
A

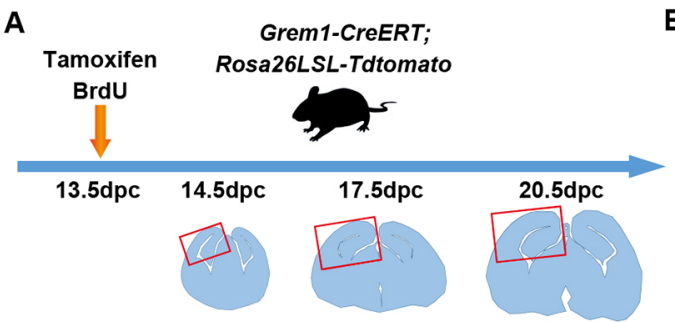

C

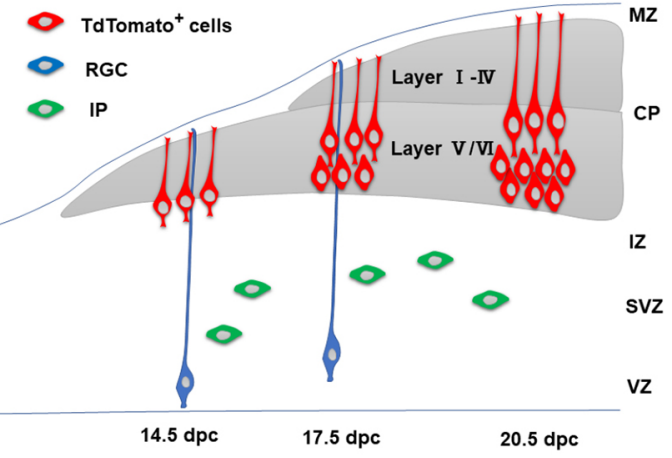

B
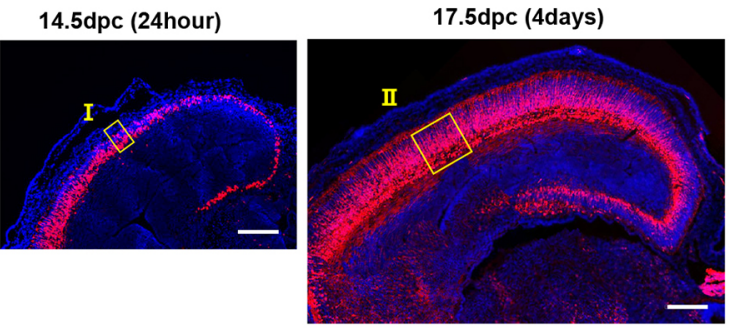

20.5dpc (7days)

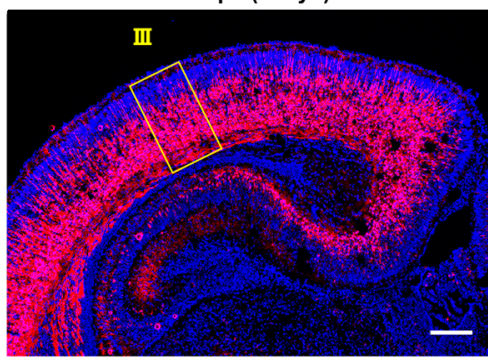

D

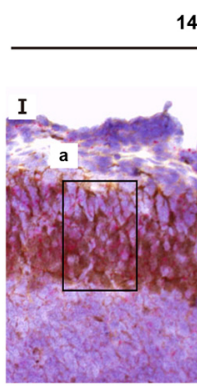

$14.5 \mathrm{dpc}$

$17.5 \mathrm{dpc}$

$20.5 \mathrm{dpc}$
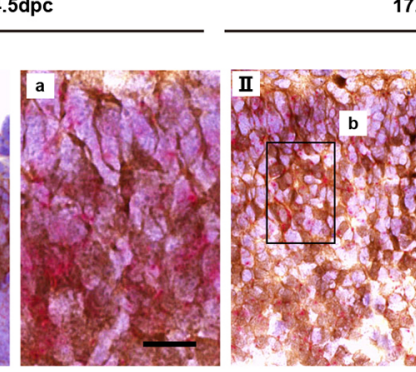

E

$\mathbf{F}$

G
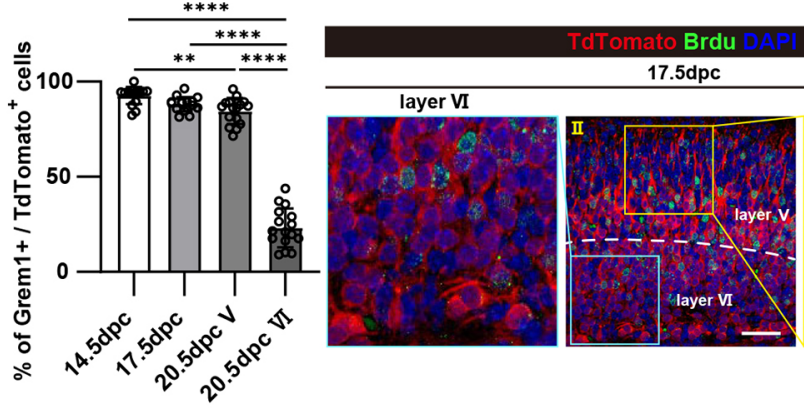

H

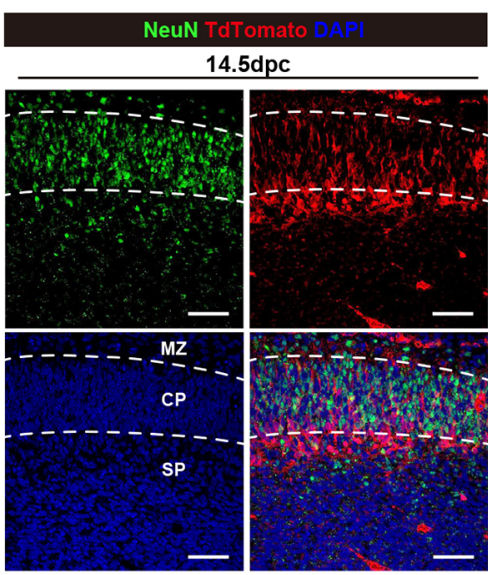

I

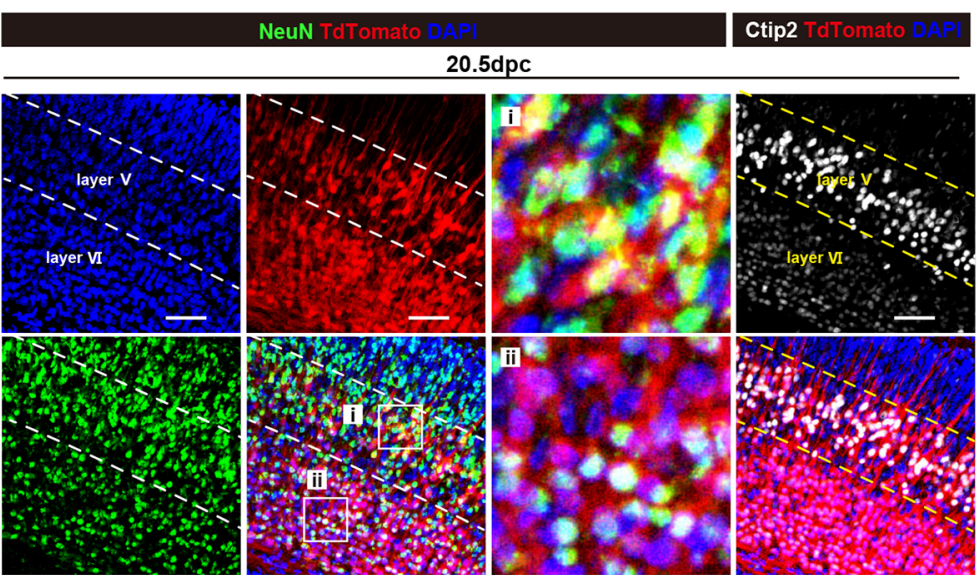

Fig. 1. See next page for legend 
Fig. 1. Grem1-expressing cells give rise to cortical neurons in the developing mouse brain. (A) Schematic showing tamoxifen and BrdU administration to Grem1creERT; Rosa26LSLTdTomato (Grem1-reporter) mice. (B) Representative images of TdTomato $^{+}$(red) cells in the telencephalon shown with red boxes in $\mathrm{A}$, at $14.5 \mathrm{dpc}$ ( $24 \mathrm{~h}$ post-induction) $17.5 \mathrm{dpc}$ ( 3 days post-induction) and $20.5 \mathrm{dpc}$ ( 6 days post-induction) in Grem1-reporter mice treated with tamoxifen at $13.5 \mathrm{dpc}$, and DAPI staining (blue). (C) Schematic showing Tdtomato traces cells differentiating to layer $V$ and VI neurons. (D) Representative images of immunohistochemical staining of boxed region from B to visualize TdTomato ${ }^{+}$cells (red) and Grem1 mRNA by ISH (green) at $14.5 \mathrm{dpc}$ (I, $24 \mathrm{~h}$ post-induction), $17.5 \mathrm{dpc}$ (II, 3 days postinduction) and $20.5 \mathrm{dpc}$ (III, 6 days post-induction) in Grem1-reporter mice treated with tamoxifen at $13.5 \mathrm{dpc}$, and DAPI staining (blue). The boxed areas were further magnified in adjacent panels. (E) Quantification of D showing the percentage of TdTomato ${ }^{+}$cells that were also Grem1 $\mathrm{RNA}^{+}$in four high-power fields (HPFs) of four biological replicates. One way ANOVA with Tukey's multiple test. (F) Representative images of immunofluorescence staining of $17.5 \mathrm{dpc}$ telencephalon from Grem1-reporter (red) mice induced with tamoxifen at 13.5 dpc: BrdU, green; DAPI, blue. (G) Quantification of F showing the percentage of TdTomato ${ }^{+}$cells that were also $\mathrm{BrdU}^{+}-$two HPFs of three biological replicates, two-tailed, unpaired $t$-test. $(\mathrm{H})$ Representative images of immunofluorescence staining of 14.5 neocortex from Grem1reporter (red) mice induced with tamoxifen at $13.5 \mathrm{dpc}$ : NeuN, green; DAPI, blue. MZ, marginal zone; CP, cortical plate; SP, subplate. (I) Representative images of immunofluorescence staining of $20.5 \mathrm{dpc}$ neocortex from Grem1reporter (red) mice induced with tamoxifen at $13.5 \mathrm{dpc}$ : NeuN, green; DAPI, blue; Ctip2, white. Layer V and VI recognized with Ctip2 are boxed in (i) and (ii), respectively. (J) Quantification of $\mathrm{H}$ showing the percentage of TdTomato ${ }^{+}$ cells that were also $\mathrm{NeuN}^{+}$in three HPF from three biological replicates. One way ANOVA with Tukey's multiple test. ${ }^{* *} P<0.01,{ }^{* * *} P<0.001,{ }^{* * * *} P<0.0001$. Data are mean \pm s.d. Scale bars: $200 \mu \mathrm{m}(B) ; 20 \mu \mathrm{m}$ (D); $50 \mu \mathrm{m}(\mathrm{F}, \mathrm{H}, \mathrm{I})$.

topological distance between different modules, i.e. how similar or different the expression of transcripts within the module are from other modules, and highlighted one module that contained 288 genes with particularly tightly correlated transcripts (magenta, Fig. S2). Network visualization of the correlation analysis showed that the majority of transcripts within this tightly correlated module are upregulated in TdTomato $^{+}$cells (magenta square, Fig. 2C). We investigated the known function of genes within this module using a hypogeometric test for gene set enrichment. Significantly enriched pathways (adjusted $P$ value $($ FDR $) \leq 0.001$ ) were related to neuronal differentiation and projection, calcium signaling (possibly associated with synapse functions) and axons (Fig. 2D). The BMP target gene Id 1 was identified as one of the central hub genes in this cluster and its expression was significantly associated with other transcripts that have functions in neuronal maturation (such as Lrrtm3, Ryr3) (Fig. 2E). Consistent with our earlier identification of Grem 1-expressing cells as committed neurons in vivo, this suggests that neuronal functions are upregulated in the Grem1-expressing TdTomato $^{+}$cells of the developing telencephalon.

\section{Grem1/GREM1 is enriched in excitatory neuronal lineage cells during brain development}

To further characterize the Grem1-expressing TdTomato $^{+}$cell population we undertook a candidate gene profiling approach using the bulk mRNA-seq data. Gene expression patterns of neuronal markers from a series of differentiation stages are shown in Fig. 3A. Immature neuronal markers, such as Dcx, Ncam1, Neurod2/6 and Tbr1, were significantly upregulated at the RNA level in TdTomato ${ }^{+}$cells in comparison with the $\mathrm{TdTomato}^{-}$cells at $14.5 \mathrm{dpc}$, whereas neural stem cell, radial glia and intermediate progenitor transcripts were significantly under-represented (FDR $<0.05$, quasi-likelihood F-test). When RGCs (marked by coexpression of Sox2 and Pax6) generate neocortical neurons, the expression of specific transcription factors can be used to identify neurons within particular cortical layers (Franco et al., 2012; Chen et al., 2005). Our bulk RNA-seq analysis showed that transcripts encoding the layer V marker Fezf2 and VI markers Tbr1 and Sox5 were significantly upregulated in Grem 1-expressing TdTomato $^{+}$ cells, whereas the RGC markers Sox 2 and Pax6 and an intermediate progenitor marker, $\mathrm{Tbr} 2$ (Eomes), were significantly downregulated (absolute value of $\log 2$ fold change $\geq 2.0, \mathrm{FDR}<0.05$ ) and did not appear to colocalize with TdTomato at $14.5 \mathrm{dpc}$ using IHC (Fig. S3).

To assist with identification of the neuronal subtype likely generated from Grem1-expressing TdTomato $^{+}$cells, we also performed Gene Set Enrichment Analysis (GSEA) on DEG transcripts between $\mathrm{TdTomato}^{+}$and $\mathrm{TdTomato}^{-}$cells. We found a significant enrichment of a glutamate secretion gene set in the DEGs [normalized enrichment score $(\mathrm{NES})=2.16, P=0.0049$ ] (Fig. 3B), whereas GABAergic and dopaminergic gene sets were not enriched $(\mathrm{NES}=-0.93, \quad P=0.75 ; \quad \mathrm{NES}=-0.69, \quad P=0.87$, respectively) (Fig. S4A). Consistent with the GSEA, glutamatergic neuron markers, such as Slc17a7 (vGlut1), Grin1 and Grin $2 \mathrm{~b}$ were significantly upregulated in Grem 1-expressing cells at $14.5 \mathrm{dpc}$ using a candidate gene approach, whereas gabaergic and dopaminergic neuron markers, such as Slc6al (GABA transporter 1), Gad1, Gad2, and Th (tyrosine hydroxylase) were significantly downregulated $(P<0.05$, Fig. S4B). Tbr1 plays a central role in the development of early-born cortical excitatory neurons and regulates the connectivity of layer VI neurons (Hevner et al., 2001). To confirm that $\mathrm{Greml}^{+}$cells generate excitatory neuronal lineages, we undertook immunohistochemical staining with Tbr1. This revealed that Grem 1-expressing TdTomato ${ }^{+}$cells at $14.5 \mathrm{dpc}$ expressed $\mathrm{Tbrl}$ at the mRNA level, but very low to no Tbr1 at the protein level; however, Grem1-TdTomato ${ }^{+}$cells did become $\mathrm{Tbrl}^{+}$neurons in cortical layer VI by $20.5 \mathrm{dpc}$ (Fig. 3C,D).

To understand the relevance of our mouse focused study to the human setting, we reanalyzed publicly available human scRNA-seq data generated from human brain at mid-gestation (22-23 weeks post-conception) (Fan et al., 2018). We categorized each human cell in the dataset as either $G R E M 1^{+}$or $G R E M 1^{-}$based on the presence or absence of GREM1 RNA-seq counts for each cell. Next, we compared the transcriptional profiles of our mouse embryonic brain Grem 1-expressing TdTomato $^{+}$cells and TdTomato ${ }^{-}$cells with the human mid-gestational brain $G R E M 1^{+}$and $G R E M 1^{-}$cells using a multidimensional scaling plot (Fig. S4C). Samples within the same group cluster tightly together, dimension 2 separates the samples along species lines, whereas dimension 1 clearly shows a similar separation of samples based on altered expression profiles depending on the Grem1/GREM1 status in mouse and human developing brain (Fig. S4C). Mapping the $G R E M 1^{+}$cells onto a tSNE plot generated from the human scRNA-seq data revealed that most GREM1-expressing cells accumulated in the excitatory neuron cluster within the human mid-gestational cortex (Fig. 3E). Reanalysis of a mouse scRNA-seq cortical neuron dataset (Yuzwa et al., 2017) also confirmed that expression of Grem1 most highly correlates with the excitatory neuron cluster marked by Foxp2 (marker of layer VI) and Ctip2 (marker of layer V/VI) and not precursor or progenitor populations (Fig. S5D). Lastly, we analyzed the expression of other BMP-signaling components in both scRNAseq datasets (Fig. S5A-D). Of the pathway antagonists similar to GREM1, sclerostin domain-containing protein 1 (SOSTDC1), $C H R D$, BMP binding endothelial regulator $(B M P E R)$ and follistatin (FST) transcripts were highly enriched in the excitatory neuron or Cajal-Retzius cell clusters, whereas $N O G$ expression correlated only with the astrocyte population in the human data that 

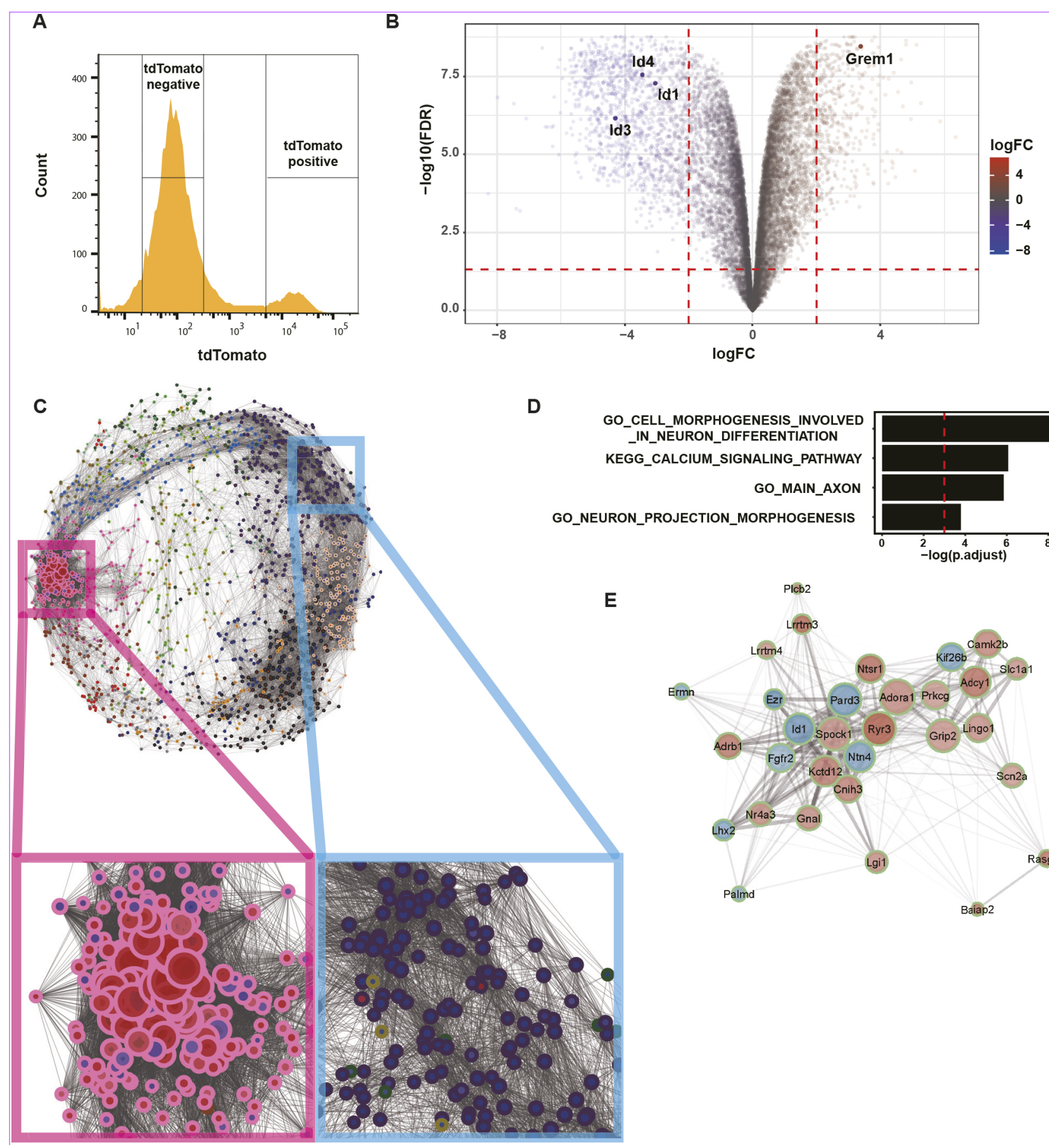

GO_CELL_MORPHOGENESIS_INVOLVED
_IN_NEURON_DIFFERENTIATION
KEGG_CALCIUM_SIGNALING_PATHWAY
GO_MAIN_AXON

GO_MAIN_AXON

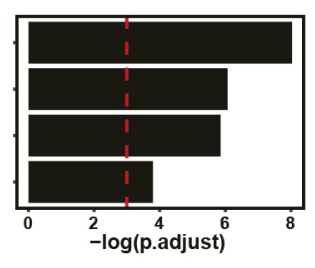

E

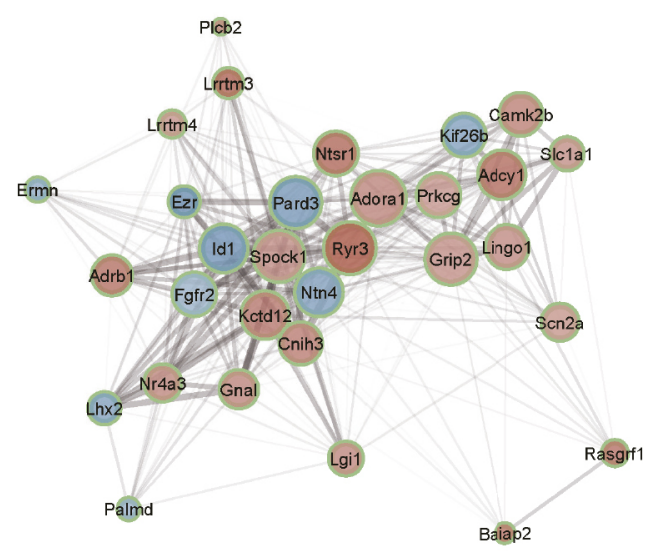

Fig. 2. Grem1-expressing cells have neuron-associated gene signatures. (A) TdTomato ${ }^{+}$and TdTomato ${ }^{-}$cells were isolated from $14.5 \mathrm{dpc}$ brains of Grem1-CreERT; R26-TdTomato mice induced with tamoxifen at $13.5 \mathrm{dpc}$. Representative FACS plot is shown, $n=5$ mice sorted for bulk RNA-seq analysis. (B) Volcano plot to show DEG between TdTomato ${ }^{+}$and TdTomato ${ }^{-}$cells from A. Grem1 was significantly upregulated, whereas BMP target genes Id1/3/4 were significantly downregulated in $\mathrm{TdTomato}^{+}$cells compared with $\mathrm{TdTomato}^{-}$cells. Absolute value of log2 fold change $\geq 2.0$, FDR<0.05. (C) Network visualization of correlated DEG modules in $\mathrm{TdTomato}^{+}$cells. Each dot represents a gene, dot perimeter color indicates module membership (see Fig. S2), dot interior color denotes upregulation (red) or downregulation (blue) of gene transcript in $\mathrm{TdTomato}^{+}$cells compared with $\mathrm{TdTomato}^{-}$, dot size indicates the magnitude of gene expression correlation to neighboring genes, lines connecting dots represent topological distance. The module containing highly correlated genes that were predominantly upregulated in TdTomato ${ }^{+}$cells is boxed in magenta. (D) Significantly enriched gene sets in magenta boxed module in C. FDR $<0.001$. (E) Genes associated with $/ d 1$, a hub gene in the magenta boxed module in C. Dot perimeter color indicates module membership (see Fig. S2), dot interior color denotes upregulation (red) or downregulation (blue) of gene transcript in TdTomato $^{+}$cells compared with TdTomato ${ }^{-}$.

contained a broad selection of neuronal and non-neuronal cell types (Fig. S5A). This analysis of BMP antagonist expression was further extended by the increased resolution of neuronal cell clusters found in the multi-time point and neuronal-restricted mouse scRNA-seq dataset, to be enriched in excitatory neurons and apical precursors/ RGCs (Fig. S5D). BMP2/7 that are antagonized by GREM1 were primarily produced by alternate RGCs and IPs/excitatory neuron populations to the Grem 1 -expressing excitatory neuron population 
A

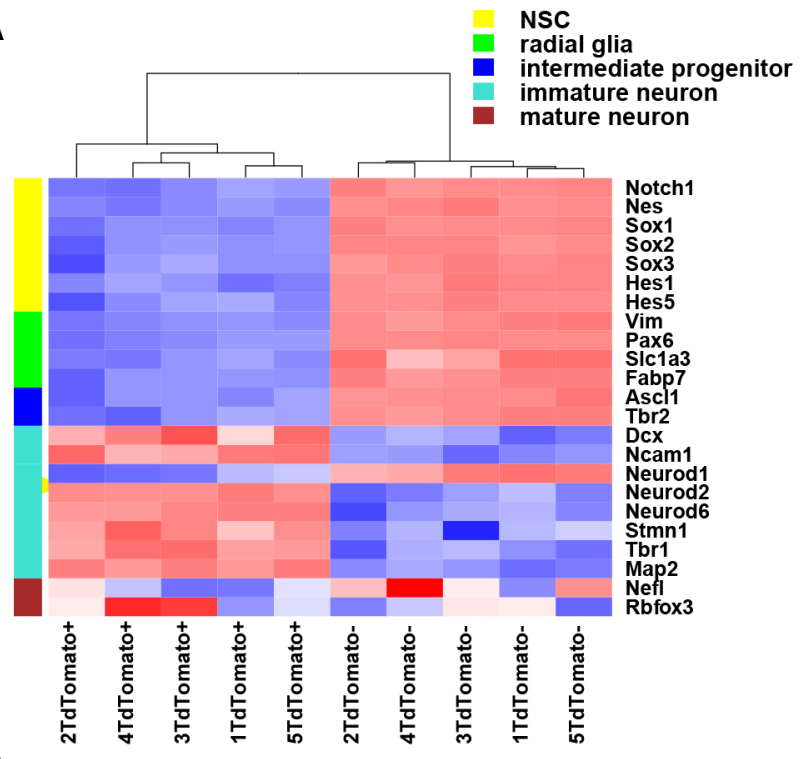

C

14.5dpc (24hours)

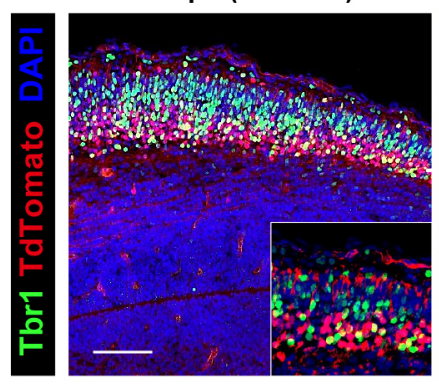

E

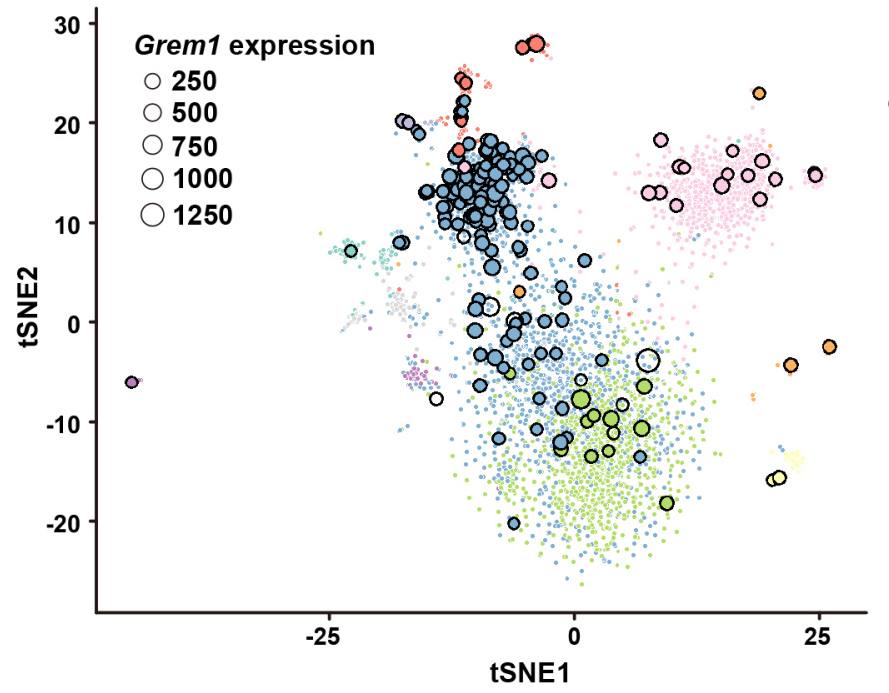

B

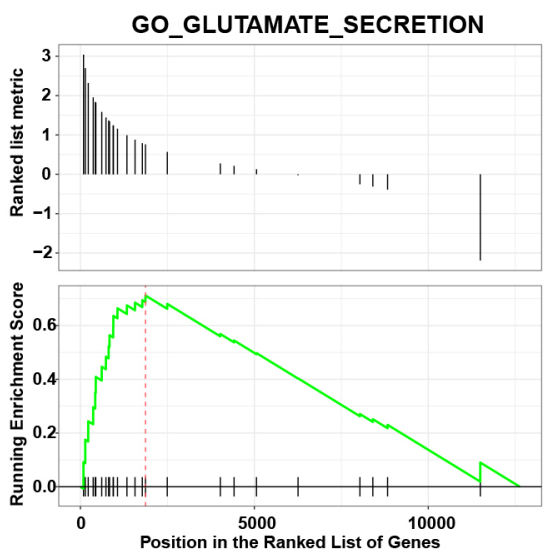

D

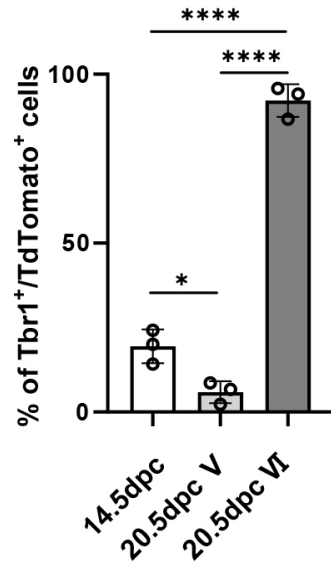

Group

Astrocyte

Blood

Cajal-Retzius

- Endothelial cell

- Excitatory neuron

- Immune

Inhibitory neuron

Microglia

NSC

Oligodendrocyte

ONA

Fig. 3. Grem1 is expressed in the glutamatergic excitatory neuron lineage cells. (A) Heatmap depicting unsupervised clustering of TdTomato ${ }^{+}$and TdTomato- cells isolated from $14.5 \mathrm{dpc}$ Grem1-reporter (red) mice induced with tamoxifen at 13.5 dpc based on expression of representative differentiation marker transcripts for neural stem cell (NSC), radial glia, intermediate progenitor, immature neuron and mature neuron. (B) GSEA for Glutamate Secretion genes between TdTomato+ and TdTomato ${ }^{-}$samples from A. NES $=2.16, P=0.0049$. (C) Representative images of immunofluorescence staining of 14.5 and 20.5 dpc telencephalon from Grem1-reporter (red) mice induced with tamoxifen at 13.5 dpc: Tbr1, green; DAPI, blue. (D) Quantification of C showing the percentage of TdTomato ${ }^{+}$cells that were also Tbr ${ }^{+}$in three representative fields from three biological replicates. One way ANOVA with Tukey's multiple test. ${ }^{*} P<0.05,{ }^{* * * *} P<0.0001$. Data are mean \pm s.d. (E) tSNE plot of human scRNA-seq dataset. GREM1-expressing cells outlined in black. Dot size represents Grem1 expression value as indicated. NA, not applicable. Scale bars: $100 \mu \mathrm{m}$. 
in the mouse developing cortex (Fig. S5D). Expression of BMP2/7 was also inversely correlated with GREMI expression in the human excitatory neuron cell population, suggesting paracrine BMP pathway regulation by these opposing factors (Fig. S5C). Expression of the BMP target genes, $I D 1 / 3 / 4$, was inversely correlated with GREM1-expression in excitatory neurons, consistent with our developmental mouse brain data and suggesting that GREMI acts locally to antagonize BMP signaling (Fig. 2B). BMP receptor type I and II expression correlated with apical precursor and proliferative RGC populations (Bmprla/1b, $A c v r 2 b)$ and excitatory neuron clusters in the mouse scRNA-seq dataset (Alk, Acvr1b/1/1c/2a,Bmpr2), suggesting that many of the neurons in the developing cortex will have the ability to respond to BMP pathway stimulation (Fig. S5A,D). This highlights the complexity of regulation of BMP signaling during development of the cortex.

\section{Grem1 promotes proliferation and neural differentiation in NSPCs ex vivo}

Next we collected NSPCs from embryonic brains of Grem $1^{\text {flox/flox }}$ mice at $14.5 \mathrm{dpc}$ and transduced the cells ex vivo with control, Creexpressing or Grem1-expressing lentivirus to generate control Grem $1^{\text {flox/flox }}$, Grem 1 ${ }^{-/-}$and Grem1 overexpressing $\left(\right.$Grem $\left.1^{O / E}\right)$ primary cultures. Endogenous Grem 1 was detected in Grem $1^{\text {flox/flox }}$ control cultures by western blot, with absent or elevated protein levels in Grem $1^{-/-}$and Grem $1^{O / E}$ cultures, respectively (Fig. 4A). There were two bands, likely because of splicing variants, consistent with previous reports (Yan et al., 2014; Koli et al., 2016). Our method does not include selection for NSPC markers or exclusion of committed lineage cells and so contains a heterogeneous mix of cells that are roughly selected for proliferation potential. Because of this, it is possible that some of this mixed population may include immature committed neurons that express Grem1, or Grem1 expression may be aberrantly upregulated in stem/progenitor cells by culture in vitro. Control Grem $1^{\text {flox/flox }}$ cultures were responsive to BMP pathway induction as determined by increased BRE-luciferase reporter activity and expression of the BMP target genes $I d 1 / 2 / 3 / 4$ following addition of recombinant human BMP2 (Fig. 4B,C; Fig. S6). This induction of BRE-reporter activity and increase in BMP target gene transcript levels was significantly enhanced in Grem $1^{-/-}$cells and attenuated in Grem $1^{O / E}$ cells (Fig. 4B,C; Fig. S6). This confirmed that Grem1 acts as an antagonist of BMP2 and suppresses downstream transcriptional targets in embryonic NSPCs. This difference was more obvious with Grem 1 overexpression than genetic deletion, possibly due to limited basal expression of Grem1 in NSPC culture.

We next employed neurosphere assays to determine the effect of Grem1 modulation on proliferation of embryonic NSPCs. Only mitogen-responsive cells proliferate to form clusters termed neurospheres, where sphere size correlates with proliferative ability (Mori et al., 2006; Reynolds and Rietze, 2005). Grem $1^{O / E}$ cells form significantly more and larger neurospheres than control Grem $1^{\text {flox/flox }}$ cells, whereas Grem $1^{-/-}$cells form significantly fewer (Fig. 4D,E). To understand this phenomenon from the aspect of cell viability, we evaluated the proliferation rates of Grem $1^{\text {flox/flox }}$, Grem $^{-/-}$and Grem1 $^{O / E}$ cultures. Overexpression of Grem1 significantly increased the rate of NSPC proliferation compared with control Grem $1^{\text {flox/flox }}$ NSPCs, whereas the number of viable Grem $1^{-/}$cells did not increase over the $72 \mathrm{~h}$ time course analyzed (Fig. 4F). Our re-analysis of the mouse neuronal cortex scRNA-seq dataset (GSE107122) showed that BMP2/7, which are antagonized by GREM1, were primarily produced by RGCs and IPs/excitatory neurons in the developing mouse cortex (Fig. S5D), suggesting BMP2/7 may be produced by the NSPCs in our in vitro system. These results suggested that Grem1 contributes to the proliferation of NSPCs. Following on from this observation, we wanted to assess the role of Grem1 in the differentiation of NSPCs to neurons, astrocytes and oligodendrocytes. When cultured in recombinant BMP2-containing differentiation media, addition of $1 \mathrm{ng} / \mathrm{ml}$ recombinant GREM1 significantly increased the number of NSPCs that differentiated into Tuj $1^{+}\left(\mathrm{Tubb3}^{+}\right)$neurons and $\mathrm{O}^{+}$ $\left(\right.$ Foxo $\left.^{+}\right)$oligodendrocyte lineage cells, and decreased the number of $\mathrm{GFAP}^{+}$astrocytes, in comparison with vehicle treated control NPSCs (Fig. 4G,H). This suggests that Grem1 may regulate the differentiation potential of NPSCs.

\section{Grem1 is required for normal cortical development}

In order to assess the functional role of Grem 1 in mouse forebrain development in vivo, we generated tissue-specific Grem 1 conditional knockout mice using the empty spiracles homeobox 1 (Emxl)-cre driver. We first verified that Emxl-cre generates efficient cre-mediated recombination in the dorsal telencephalon at $14.5 \mathrm{dpc}$ by visualizing TdTomato $^{+}$cells in reporter Emxl-cre; Rosa26LSLTdtomato mouse brains (Fig. S7A). Next we confirmed that Emxl-cre; Grem1 flox/flox (Greml conditional knockout, Grem $1^{c K O}$ ) mice had a significant reduction in Grem 1 RNA in the developing forebrain compared with $\mathrm{cre}^{-}$littermate controls by ISH at $14.5 \mathrm{dpc}$ (Fig. 5A) and real time RT-PCR at P0 (Fig. S7B). Grem $1^{\text {cKO }}$ mice were viable and fertile. To determine the mechanistic effect of loss of Grem 1 we directly analyzed BMP pathway activity by immunohistochemical staining for the downstream pathway effectors, phospho-SMAD1/5/8. The number of Ctip2 ${ }^{+}$layer V/VI cells with active BMP signaling (phospho-SMAD1/5/8 ${ }^{+}$) was significantly increased in Grem $1^{\text {cKO }}$ mice in comparison with Grem $1^{\text {flox/flox }}$ littermate controls (Fig. 5B, $\mathrm{C}$; Fig. S7C). To examine the morphological consequences of conditional Grem1 loss in the developing mouse brain we performed Nissl staining on tissue samples from Grem $^{c K O}$ and $\mathrm{cre}^{-}$littermate controls. At 10 weeks of age, total cortical thickness was significantly reduced in Grem $1^{c K O}$ mice in comparison with Grem $1^{\text {flox/flox }}$ littermate controls both in males and females, owing to significantly thinner cortical layers V and VI as marked by Ctip2 and Foxp2 (Fig. 5D,E; Fig. S7D,E,F). Cellular density was also lower in Grem $1^{\text {cKO }}$ mice in comparison with Grem $1^{\text {flox/flox }}$ littermate controls (Fig. S7G,H). The number of $\mathrm{Ki} 67^{+}$proliferative cells was unchanged both at $14.5 \mathrm{dpc}$ and $20.5 \mathrm{dpc}$ between Grem $^{c K O}$ and Grem $1^{\text {flox/flox }}$ littermate controls (Fig. S7I,J,K,L). Also the number of $\mathrm{Tbr}^{+}$cells was unchanged at $14.5 \mathrm{dpc}$ (Fig. S7I,J). In contrast, the number of cleaved caspase $3^{+}$cells was increased in Ctip2 ${ }^{+}$ layers of Grem $1^{c K O}$ mice in comparison with Grem $1^{\text {flox/flox }}$ littermate controls at $20.5 \mathrm{dpc}$, but was not different at $14.5 \mathrm{dpc}$ (Fig. 5F,H; Fig. S7M). This increase in apoptotic cells marked by cleaved caspase 3 likely explains the significantly thinner Ctip2 ${ }^{+}$layer we observed in Grem $1^{c K O}$ animals compared with Grem $1^{\text {flox/flox }}$ littermate controls; however, our analyses have not ruled out potential changes in cell proliferation or cell fate decisions between the 14.5 and $20.5 \mathrm{dpc}$ time points examined (Fig. 5G). Conversely, the other predominant region of Emxl-cre driver activity, the hippocampus, which has important functions in memory for navigation pertinent to our behavioral testing, displayed morphology and cell density that did not appear to be different to normal on microscopic inspection (Fig. S8A).

Our earlier transcriptional network analysis from bulk RNA-seq data identified an $I d I$-associated gene cluster that was differentially 
A

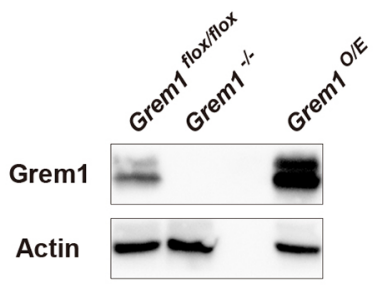

B

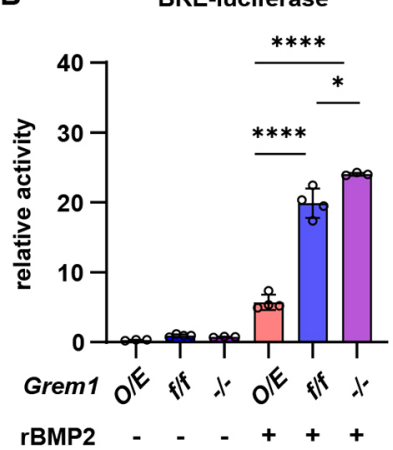

C

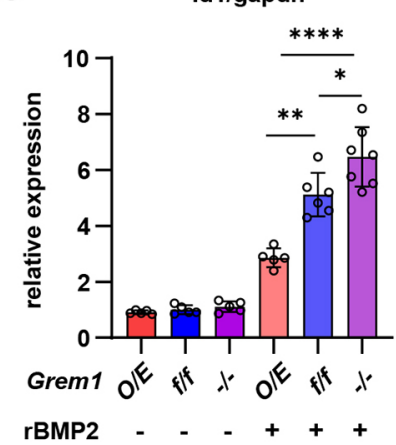

D

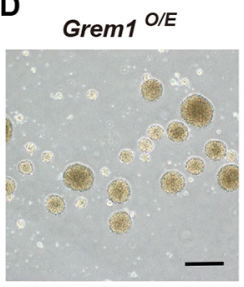

Grem1 flox/flox

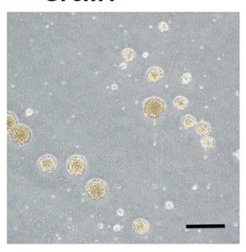

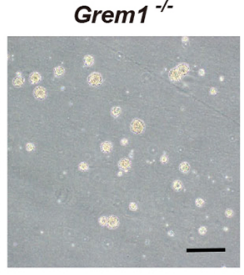

G
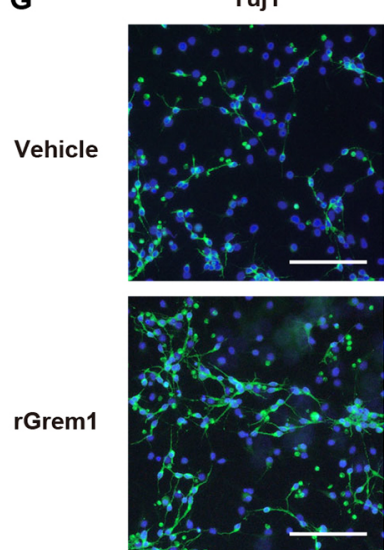

neurosphere number

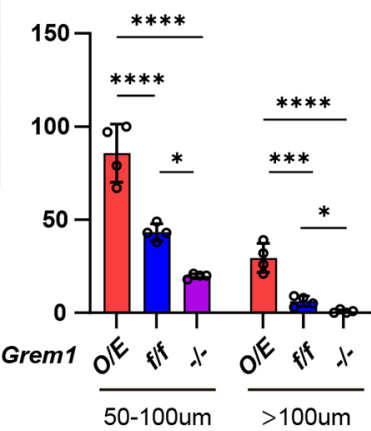

GFAP
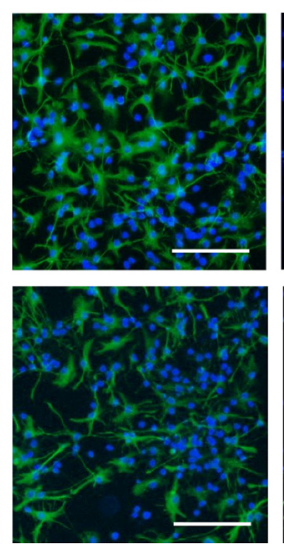

O4
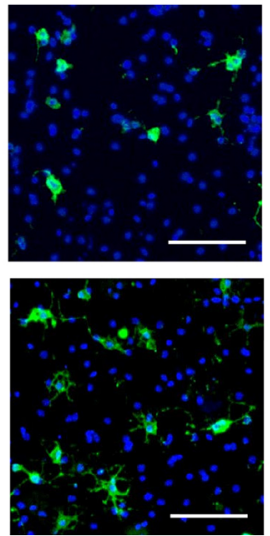

F

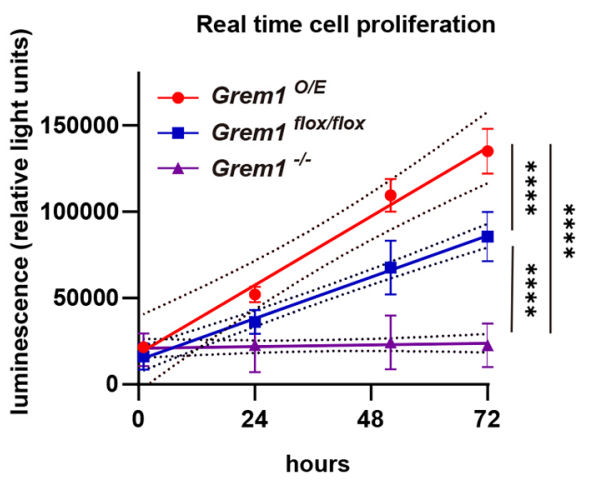

H

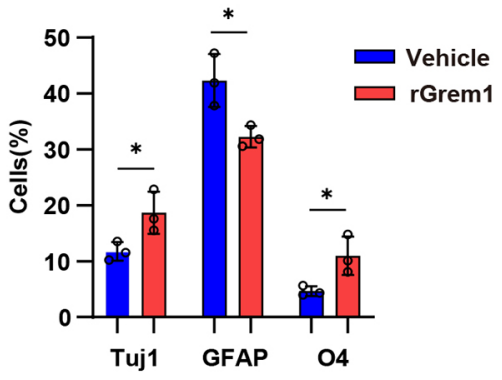

Fig. 4. Grem1 promotes proliferation and neuronal differentiation in neural stem/progenitor cells ex vivo. (A) Detection of Grem1 protein levels in control Grem $1^{\text {flox/flox }}$, Grem $1^{-1-}$ and Grem1 ${ }^{O / E}$ NSPC by western blot. (B) BRE-reporter activity relative to internal control reporter. Data obtained from four independent experiments each performed in triplicate. One way ANOVA with Tukey's multiple test. (C) BMP target gene Id1 mRNA expression normalized to

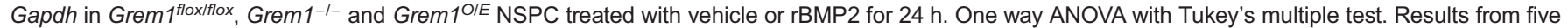

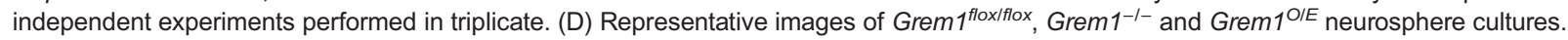

(E) Quantification of neurosphere number and size from $D$ in four representative fields from four independent experiments. One way ANOVA with Tukey's multiple test. $(F)$ Cell proliferation normalized to $0 \mathrm{~h}$ time point for each culture. Results from three independent experiments each performed in triplicate and analyzed using linear regression. (G) Representative images of immunofluorescence staining to identify Tuj $1^{+}$neurons, $\mathrm{GFAP}^{+}$astrocytes and $\mathrm{O}^{+}$ oligodendrocytes in differentiated cultures from wild-type NSPCs treated with vehicle or rGrem1. (H) Quantification of G, showing the percentage of marker ${ }^{+}$ cells in three representative fields from three independent experiments. Two-tailed, unpaired $t$-test. Data are mean \pm s.d. ${ }^{*} P<0.05,{ }^{* *} P<0.01,{ }^{* * *} P<0.001$, ${ }^{* * *} P<0.0001$. Scale bars: $100 \mu \mathrm{m}$.

regulated in the Grem 1-expressing TdTomato ${ }^{+}$cells from the cortex at $14.5 \mathrm{dpc}$ (Fig. 2E). From this cluster, Lrrtm 3 and Ryr3 were selected for further analysis because of their known functions in synapse development (Balschun et al., 1999; Um et al., 2016; Roppongi et al., 2017; Futagi and Kitano, 2015; Linhoff et al.,
2009). The expression of these two transcripts was significantly downregulated in the cortex of Grem $1^{c K O}$ mice compared with littermate controls at P10 (Fig. S8B). This provides further evidence of the relationship between Grem 1 expression and these genes. To examine the effect of Grem1 deletion on differentiation, transcript 
A

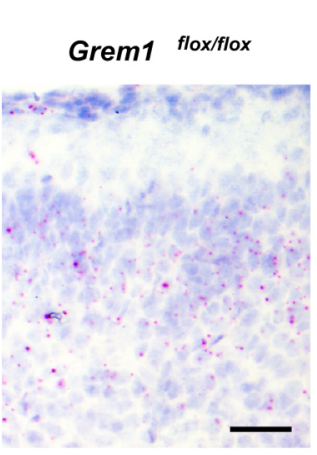

D

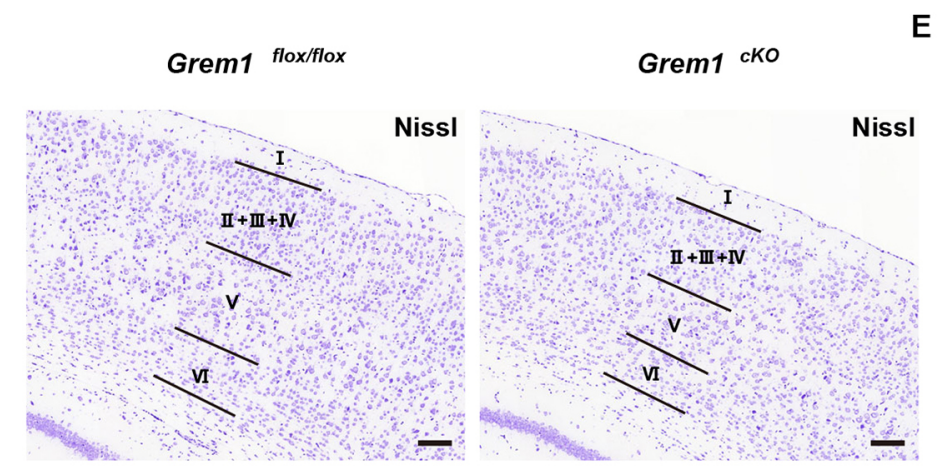

F
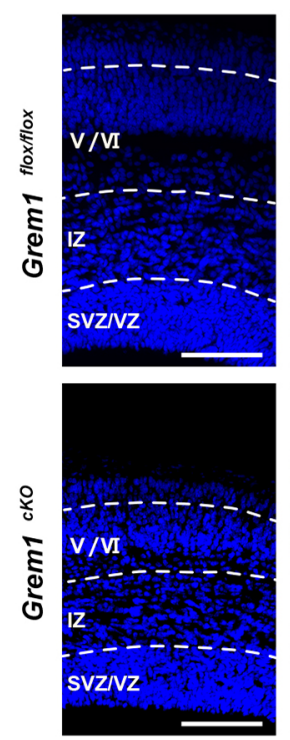

I

$$
\begin{aligned}
& \square \text { Grem1 }{ }^{\text {flox/flox }} \\
& \square \text { Grem1 } 1^{\text {cko }}
\end{aligned}
$$

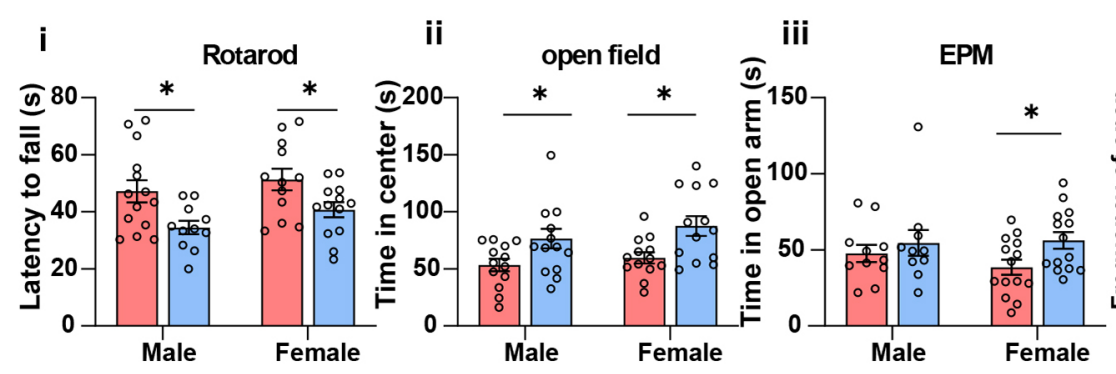

Cho cleaved-caspase 3
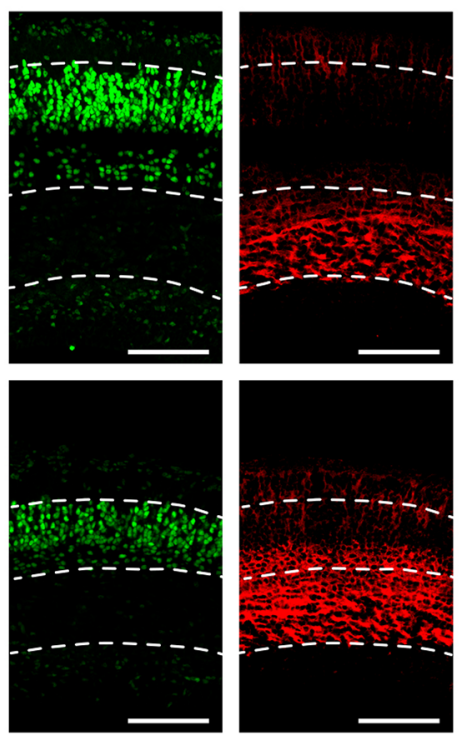

B

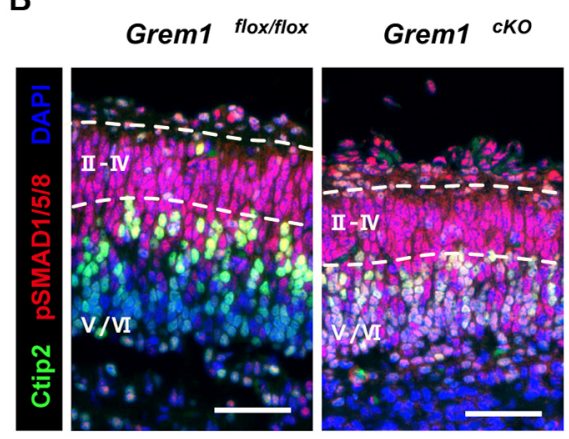

C

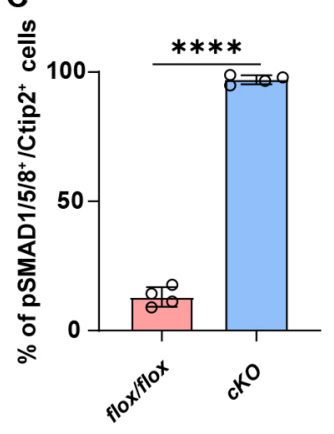

E $\left.\quad{ }^{1000}\right]$ Male
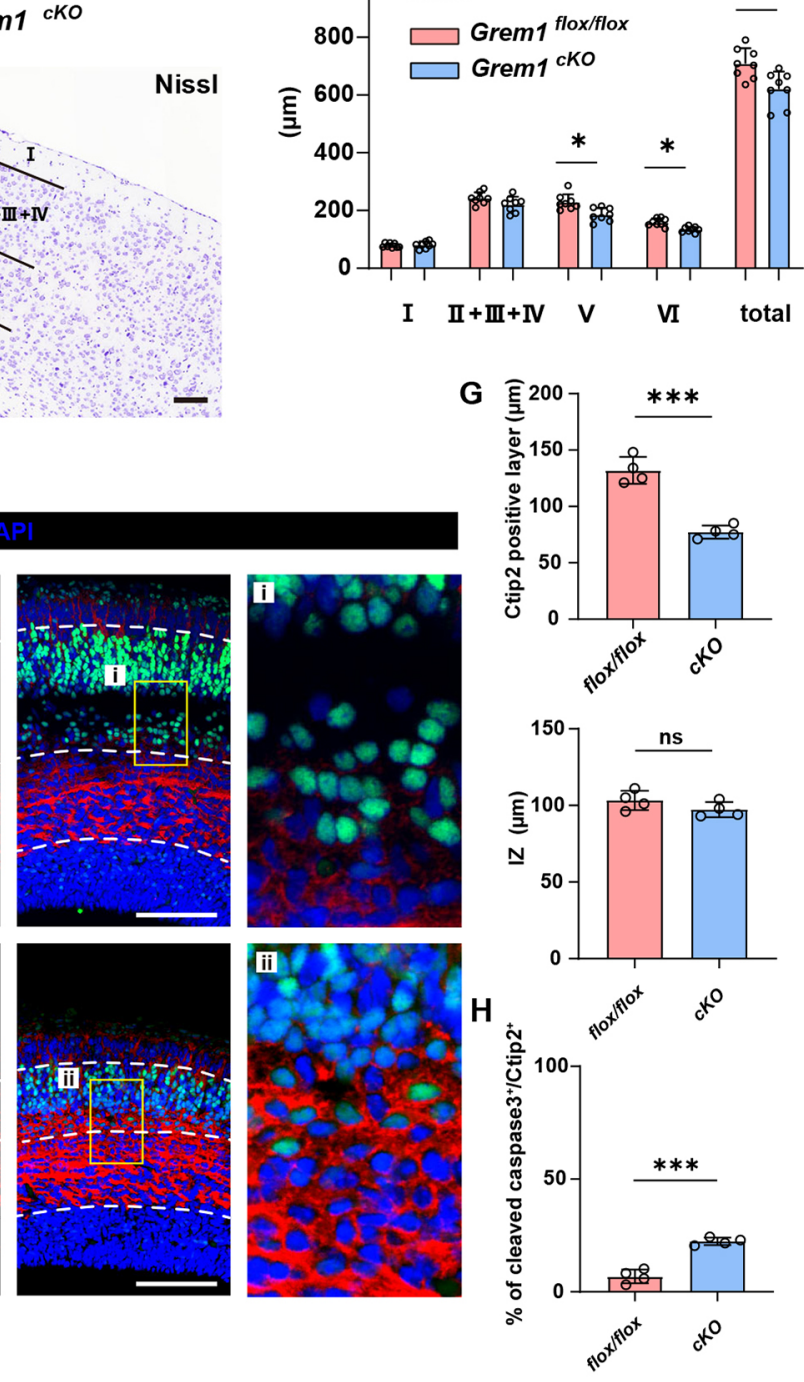

iv $\quad$ EPM

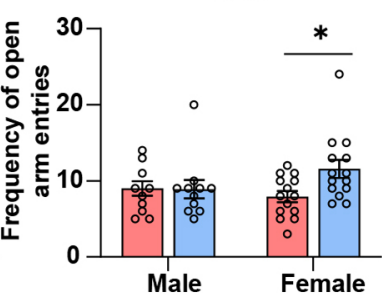

Fig. 5. See next page for legend. 
Fig. 5. Cortical development, motor balance and fear is impaired in Grem1 conditional knockout mice: Grem1 is required for normal cortical development. (A) Representative Grem1 expression in the dorsal telencephalon of Grem $1^{c K O}$ mice and Grem $1^{\text {flox } / f l o x}$ littermate controls by ISH at $20.5 \mathrm{dpc}$. $n=3$. (B) Representative images of immunofluorescence staining of cortex of Grem $1^{1 K O}$ mice and littermate controls at $20.5 \mathrm{dpc}$ with layer $\mathrm{V}$ and VI showing marker Ctip2 (green), phosphorylated SMAD1/5/8 (red) and DAPI (blue) (see also Fig. S7C). (C) Quantification of phosphorylated SMAD $1 / 5 / 8^{+}$cells in Ctip2 ${ }^{+}$cells in (B). $n=4$, two-tailed, unpaired $t$-test. (D) Representative histological images of cortical layers of Grem $1^{\text {cKO }}$ mice and Grem $1^{\text {flox/flox }}$ littermate controls using Nissl staining at 10 weeks of age. (E) Quantification of cortical layer thickness from D compared in eight pairs of male Grem $1^{\text {CKO }}$ mice and littermate controls at 10 weeks of age. Male, $n=7$ control and $n=7 \mathrm{Grem} 1^{1 K O}$, two-tailed, unpaired $t$-test. $(\mathrm{F})$ Representative images of immunofluorescence staining of cortex of Grem $1^{1 \mathrm{KO}}$ mice and littermate controls at $20.5 \mathrm{dpc}$ with layer $\mathrm{V}$ and $\mathrm{VI}$ showing marker Ctip2 (green), cleaved caspase 3 (red) and DAPI (blue). $n=4$. High magnification of boxed areas is shown in right panels. (G) Quantification of Ctip2 ${ }^{+}$layer V/VI and intermediate zone thickness from $\mathrm{F}$ compared in four pairs of Grem $1^{\mathrm{c} K O}$ mice and littermate controls at $20.5 \mathrm{dpc}$, two-tailed, unpaired $t$-test.

$(\mathrm{H})$ Quantification of cleaved caspase $3^{+}$cells in Ctip2 ${ }^{+}$cells in G. $n=4$, two-tailed, unpaired $t$-test. (I) Behavioral testing was performed to compare Grem $1^{\text {cKO }}$ and Grem $1^{\text {flox } / \text { flox }}$ mice using age and sex matched littermates at 7-10 weeks of age. (i) Rotarod test latency to fall. Male, $n=14$ control and $n=11$ Grem $1^{\text {cKO}}$; Female, $n=12$ control and $n=13 \mathrm{Grem} 1^{\text {cKO }}$, two-tailed, unpaired $t$-test. (ii) Open field test, cumulative duration spent in the center area. Male, $n=13$ control and $n=14 \mathrm{Grem} 1^{c K O}$; Female, $n=13$ control and $n=13$ Grem $1^{c K O}$, two-tailed, unpaired $t$-test. (iii,iv) Elevated plus maze test. (iii) Cumulative duration spent in open arms. (iv) The number of entries to open arms. Male, $n=12$ control and $n=11$ Grem $1^{\text {cKO}}$; Female, $n=14$ control and $n=14 \mathrm{Grem} 1^{\mathrm{cKO}}$, two-tailed, unpaired $t$-test. Data are mean \pm s.e.m. ${ }^{*} P<0.05,{ }^{* * *} P<0.001$, ${ }^{* * * *} P<0.0001$. Scale bars: $100 \mu \mathrm{m}$.

levels of Slc17a7 for excitatory neurons, Gfap for astrocytes and myelin-associated glycoprotein (Mag) for oligodendrocytes were also assessed by qPCR. Although Slc17a7 was significantly decreased in Grem $1^{c K O}$ samples, there was no significant difference in Gfap or Mag (Fig. S8B).

\section{Motor coordination and fear responses were impaired in Grem1 ${ }^{\text {cKo }}$ mice}

To assess the functional consequences of embryonic Grem 1 deletion in the mouse forebrain, we undertook behavioral testing with Grem $1^{c K O}$ mice in comparison with Grem $1^{\text {flox/flox }}$ littermate controls. We compared motor balance using the Rotarod test. Latency to fall was significantly shorter in Grem $1^{c K O}$ mice both for males and females, suggesting an impaired motor balance following loss of Grem1 (Fig. 5I). During the open field test used to assess exploratory behaviors, both male and female Grem $1^{c K O}$ mice spent significantly more time in the central area away from the walls than littermate controls (Fig. 5I). The total distance moved and velocity of movement were similar between Grem $1^{c K O}$ and littermate controls (Fig. S8C,D). Behavioral testing using an elevated plus maze also indicated that female Grem1 ${ }^{c K O}$ mice spent significantly more time in the open maze arms and entered into the open arms more frequently than littermate controls (Fig. 5I). Both of the exploratory behavior tests indicated that conditional loss of Grem1 leads to reduced anxietylike behavior. Lastly, we used the Y maze test to assess the shortterm memory of Grem $1^{c K O}$ and littermate controls. We observed no difference in the number of entries made to the novel arm of the $\mathrm{Y}$ maze between groups (Fig. S8E,F), suggesting that Grem 1 expression is not required for short-term memory function.

\section{DISCUSSION}

The spatiotemporal regulation of BMP signaling in brain development is poorly understood. Here, we focus on the expression and function of the BMP antagonist Grem1 during brain development, using transgenic lineage tracing, gene expression, in vitro culture and conditional knockout approaches.

In the developing mouse brain Greml is first expressed at $13.5 \mathrm{dpc}$ in committed neurons that will differentiate into layer $\mathrm{V}$ and VI neurons by 20.5 dpc (Fig. 1I; Fig. S1A,C). Transcriptomic profiling using our mRNA-seq data from embryonic Grem1reporter mouse brains and publicly available mouse and human developmental brain scRNA-seq data suggests that Grem1/GREM1 expression is primarily associated with markers of glutamatergic excitatory neuronal lineages, rather than GABAergic or dopaminergic neurons or other cell types of the developing brain (Fig. 3B,E; Fig. S4A,B). Consistent with this, Grem1 was not expressed in the ganglionic eminences, from which GABAergic interneurons originate; nor were GABAergic transcripts upregulated or enriched by GSEA in Grem 1-expressing TdTomato $^{+}$cells in the dorsal telencephalon using bulk RNA-seq analysis (Fig. S4A,B). Likewise Grem1-lineage-traced cortical neurons expressed the excitatory marker Tbr1 (Fig. 3C). Greml-expression labels and generates important excitatory neuronal lineages, i.e. pyramidal neurons, which play high-level cognitive functions in the neocortex. Grem 1 expression is required for the correct development of earlyborn excitatory neurons of the neocortex (Figs 1I and 5D-H). Although we have not directly analyzed the proportion of excitatory and inhibitory neurons in Grem $1^{c K O}$ animals here, misregulation of BMP signaling might affect the balance between excitatory and inhibitory neuronal activities, which has been implicated in neurodevelopmental disorders (Dani et al., 2005).

Our analysis of scRNA-seq data from human midgestational cortex (22-23 weeks post-conception) and from mouse embryonic cortex suggests that there is coordinated regulation of BMP signaling during development, but that there may be also be functional redundancies (Fig. S5A,D). Nog is the most extensively studied of the BMP antagonists in the CNS, in which it is a recognized neural inducer during gastrulation (Lamb et al., 1993). Addition of recombinant Nog to neural stem cells promotes neuronal and suppresses astrocytic differentiation in vitro (Mikawa and Sato, 2011). In our study, we confirmed that Grem1 acts as a BMP antagonist in embryonic NSPC cultures, suppressing downstream BMP transcriptional targets (Fig. 4B,C; Figs S5C, S6). Although Grem1 is expressed by immature but committed neurons, rather than $\mathrm{Sox}^{+}$or $\mathrm{Pax}^{+}$neural stem or precursor populations in vivo (Fig. S3), this in vitro study was useful to simulate the role of secreted Grem1. Similar to Nog, we determined that Grem1 promotes proliferation and neural differentiation, at the expense of astrocytic lineages in vitro (Fig. $4 \mathrm{G}, \mathrm{H}$ ). This is consistent with a previous report that found Grem1 promotes proliferation and suppresses astrocyte differentiation of cancer stem cells in glioblastoma (Yan et al., 2014). Grem1 ${ }^{c K O}$ mice showed decreased Slc17a7 mRNA expression, but no significant change in glial cell differentiation, possibly due to the redundant BMP regulatory effects by other antagonists in vivo.

Our histological analyses identified that the cortex (particularly neocortical layers V and VI) of $G r e m 1^{c K O}$ animals is significantly thinner, with less cells, in comparison with littermate controls, with no obvious morphological changes in the hippocampus. In contrast to our NSPC in vitro data (Fig. 4D-F), we did not observe a significant decrease in the number of $\mathrm{Ki} 67^{+}$proliferative cells or $\mathrm{Tbr}^{+}$ intermediate progenitors in $G r e m 1^{c K O}$ animals at $14.5 \mathrm{dpc}$ or 20.5 dpc (Fig. S7I-L). However, it is possible that progenitors may have altered proliferation or differentiation in Grem $^{c K O}$ animals between the time points analyzed. Still, the number of cleaved caspase 
$3^{+}$apoptotic cells was increased in cortical layers V/VI in the absence of Grem1 in Grem1 $1^{c K O}$ animals at $20.5 \mathrm{dpc}$, whereas Ctip2 and cleaved caspase 3 were not changed at 14.5 dpc (Fig. 5F,H; Fig. S7M). This suggests that apoptosis was increased in Grem $1^{c K O}$ after $14.5 \mathrm{dpc}$, which could contribute to the thinner deep cortical layers in these animals after birth in comparison with littermate controls.

Although secreted Grem1 can influence the upper as well as deep-layer neurons, we did not observe any noticeable changes to downstream BMP pathway activity in the upper layer neurons of Grem $1^{c K O}$ mice in comparison with Grem $1^{\text {flox/flox }}$ littermate controls as indicated by phospho-SMAD $1 / 5 / 8$ staining (Fig. 5B,C; Fig. S7C). We did however observe an increase in phosphoSMAD1/5/8 staining in Ctip2 ${ }^{+}$deep-layer neurons in Grem $1^{\text {cKO }}$ mice in comparison with Grem $1^{\text {flox/flox }}$ littermate controls. BMP transcriptional targets were significantly downregulated in Grem1expressing TdTomato $^{+}$cells in vivo (Fig. 2B) and Grem $1^{+}$excitatory neurons in the human embryonic cortex (Fig. S5C). An upper layer neuron marker, $C u x 1$, and expression of BMP receptors Bmpr $1 a / 1 b$ and $A v c r 2 b$ correlated with apical precursor and proliferative RGC populations, whereas deep-layer neuron markers Foxp2 and Ctip2, and BMP receptors Alk, Acvrlb/1/1c/2a and Bmpr2 expression correlated with excitatory neuron or newborn neuron clusters in the mouse scRNA-seq cortical neuron dataset (Yuzwa et al., 2017) (Fig. S5D). This suggests that BMP receptors are expressed both in upper and deeper layer cortical neurons, although with specific celltype expression patterns for each receptor. Conversely, BMPs are produced by endothelial cells, astrocytes and excitatory neurons in human (Fig.S5B). In the mouse cortex, BMPs are produced by RGCs and IPs/excitatory neuron populations, according to single cell analysis of neuronal lineage cells (Fig. S5D), and also from the meninges (Choe et al., 2012) (Fig. S5E). Although Grem1 is secreted, it usually acts on nearby or adjacent cells (Worthley et al., 2015). This could explain why downstream SMAD signaling is most affected in deep-layer neurons in which Grem1 is predominantly expressed. Together these results suggest that Grem1-expressing cells are fated to become neurons via antagonism of potential paracrine BMP signals (Fig. S5E) (Furuta et al., 1997; Imura et al., 2008; Choe and Pleasure, 2018).

Layer V neurons target the spinal cord, cerebellum, striatum and the thalamus (Shipp, 2007) and play roles in movement preparation, movement guidance and the execution of well-timed movements (Baker et al., 2018; Li et al., 2015). The observed decrease in layer V neurons would lead to impaired preparation for and coordination of movement in Grem $1^{c K O}$ animals and explain the motor balance defect in these animals in comparison with litter mate controls. Emerging evidence suggests that layer VI neurons play a central role in modulating thalamic and cortical neurons during sensory processing (Wang et al., 2016). Our behavioral testing confirmed that loss of Grem 1 expression significantly impaired fear sensitivity in Grem $1^{c K O}$ animals, compared with littermate controls, consistent with a deficiency in layer VI neurons with roles in sensory connections. BMP signaling is known to promote synaptogenesis (Xiao et al., 2013; Shen et al., 2004) and altered synaptogenesis can affect both fear sensitivity and motor abilities (Ha et al., 2016; Wood and Shepherd, 2010). Network analysis of DEG between Grem1expressing TdTomato $^{+}$and TdTomato ${ }^{-}$cells from the developing mouse dorsal telencephalon identified a gene cluster of inter-related transcripts in which the BMP target gene, Id1, acts as a hub. Genes in the cluster play roles in neuron morphogenesis, synapse and axon maturation (Fig. 2D,E). For example, Lrrtm3/4 encode synapse organizing proteins crucial for the development and function of excitatory synapses (Um et al., 2016; Roppongi et al., 2017; Linhoff et al., 2009). Likewise, Ryr3 encodes a member of a family of receptors that shape synaptic transmissions(Balschun et al., 1999) by amplifying spike-driven calcium signals in presynaptic terminals, and consequently enhancing the efficacy of transmitter release (Futagi and Kitano, 2015). In Grem $1^{c K O}$ animals, the mRNA expression of Lrrtm3 and Ryr3 was significantly decreased in comparison with littermate controls (Fig. S8B). This is possibly because of a loss of excitatory deep-layer neurons in the gross cortex tissue samples from Grem $1^{c K O}$ animals compared with Grem $1^{\text {flox }}$ flox littermate controls, rather than indicating a direct role for Grem1 in the regulation of synaptogenesis. However the potential role for Grem1 and other BMP modulators in neuron morphogenesis, synapse and axon maturation in the developing cortex warrants further interrogation.

In summary, this is the first study to reveal the important function of Grem 1 in cortical development. Grem1 is expressed and essential for survival of committed deep-layer cortical neurons. In the future, Grem1 may hold value beyond understanding the cellular biology of brain development and function as we develop new approaches to help tackle complex neurodevelopmental and neurological diseases.

\section{MATERIALS AND METHODS \\ Mice}

Grem1-CreERT transgenic mice (Worthley et al., 2015) were crossed with R26-LSLTdTomato mice [B6.Cg-Gt(ROSA)26Sor tm 14(CAG-tdTomato)Hze $/ \mathrm{J}$; JAX 007914] to generate a tamoxifen-induced Grem1 reporter line. Pregnant dams were administered $6 \mathrm{mg}$ of tamoxifen by oral gavage to induce embryonic Greml-tracing, with some dams bearing $13.5 \mathrm{dpc}$ embryos also intraperitoneally injected with BrdU (Roche, $40 \mathrm{mg} / \mathrm{kg}$ ) to track cell division. Grem $1^{\text {flox/flox }}$ mice (Gazzerro et al., 2007) were crossed

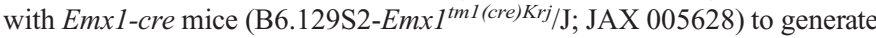
Emxl-cre-mediated Greml conditional knockout mice (Emxl-cKO). The line was maintained by crossing Emxl $1^{\text {cre/+ }}$; Greml $1^{\text {flox/flox }}$ mice with Grem $1^{\text {flox/flox }}$ mice.

All mice were on the C57BL6/J background and experimentation was conducted following approval by South Australian Health and Medical Research Institute (SAHMRI) Animal Ethics Committee (approval number SAM284) in accordance with the Australian Code for the Care and Use of Animals for Scientific Purposes (8th edition).

\section{Preparation of single cell suspensions and flow cytometry}

Pregnant Grem1creERT; Rosa26LSLTdTomato mice were administered tamoxifen at $13.5 \mathrm{dpc}$. At $14.5 \mathrm{dpc}$, dorsal telencephalons of the embryos were dissected in cold phosphate-buffered saline (PBS) and the meningeal membranes were removed. Neurocult Enzymatic Dissociation kit (Stemcell Technologies) was used for cell dissociation according to the manufacturer's protocol. Dissociated cells were resuspended in fluorescence-activated cell sorting (FACS) buffer containing DAPI $(0.5 \mu \mathrm{g} / \mathrm{ml})$. Sorting and analyses were carried out on a FACS Fusion flow cytometer (Becton-Dickinson). Dead cells were excluded by gating on forward and side scatter and by eliminating $\mathrm{DAPI}^{+}$events. The cells harvested from cre ${ }^{-}$littermate control mice were used to set background fluorescence levels. Viable cells were sorted into FACS buffer, collected via centrifugation (300 g) and resuspended in Trizol (Invitrogen) for RNA extraction.

\section{RNA extraction and mRNA-seq}

RNA was extracted from sorted cells in Trizol according to the manufacturer's protocol with the exception of addition of glycogen $(20 \mu \mathrm{g} / \mu \mathrm{l})$ and isopropanol precipitation overnight at $-80^{\circ} \mathrm{C}$ to maximize yield. RNA quality and quantity were analyzed using a NanoDrop and TapeStation (Thermo Scientific). Total RNA was converted to strandspecific Illumina-compatible sequencing libraries using the Nugen Universal Plus mRNA-Seq library kit from Tecan (Mannedorf) as per the manufacturer's instructions (MO1442 v2). Briefly, $500 \mathrm{ng}$ of total RNA was polyA selected and the mRNA fragmented before reverse transcription and 
second strand cDNA synthesis using dUTP. The resultant cDNA was end repaired before the ligation of Illumina-compatible barcoded sequencing adapters. The cDNA libraries were strand selected and PCR amplified for 12 cycles before assessment by Agilent Tapestation for quality and Qubit fluorescence assay for quantity. Sequencing pools were generated by mixing equimolar amounts of compatible sample libraries based on the Qubit measurements. Sequencing of the library pool was performed with an Illumina Nextseq 500 using single read 75 bp (v2.0) sequencing chemistry.

\section{Bioinformatic analysis RNA-seq data processing}

Fastq files from the sequencing run were subjected to quality controls with FastQC version 0.11.3. Raw reads with low quality were removed using Trim Galore alignment (Phred score less than 28 and/or reads contains adaptor sequences). After trimming, all bases with low quality scores and adaptor sequences were removed. The trimmed reads were mapped to Ensembl mouse genome (GRCm38) with STAR 2.4.2a. No more than one base mismatch was allowed. Only uniquely mapped reads were retained. Option -quantMode was enabled to generate gene level quantification. The counts files were then merged into an expression table for downstream differential expression analysis.

\section{Differential expression analysis}

DEG between the TdTomato $^{+}\left(\right.$Grem $\left.^{+}\right)$and TdTomato $^{-}\left(\right.$Grem $\left.^{-}\right)$ populations were analyzed with edge $\mathrm{R}$ packages in $\mathrm{R}$ version 3.6.0 (Robinson et al., 2010). Reads were inspected through a multidimensional scaling plot and outliers were removed. Weighted trimmed mean of $\mathrm{M}$ values (TMM) normalized counts were $\log 2$-transformed and counts per million (CPM) obtained. Paired comparisons between the TdTomato ${ }^{+}$ $\left(\right.$ Grem $\left.^{+}\right)$and TdTomato $^{-}\left(\right.$Grem $\left.^{-}\right)$populations were performed. DEG reported as significant were selected by requiring both adjusted $P$-value $($ FDR $) \leq 0.05$ and absolute value of $\log 2$ fold change $\geq 2.0$.

\section{Supervised Weighted Gene Correlation Network Analysis (WGCNA)}

Only DEGs were used for subsequent network analysis. The gene network was constructed using the $\mathrm{R}$ package supervised weighted gene correlation network analysis (WGCNA) following the procedure previously described (Langfelder and Horvath, 2008). After the low expression genes (FPKM $<1$ ) had been filtered out from all gene expression libraries, Pearson's correlation-based adjacency was calculated on the basis of pairwise correlations of gene expression within TdTomato $^{+}\left(\right.$Greml $\left.^{+}\right)$samples. Topological overlap of the correlations was used to weight the edges of the correlation network. The higher the weight, the stronger the interaction between two genes. Connectivity for a single gene was calculated as the sum of weights relative to the rest of the genes, and the top 5\% of genes with the highest connectivity in the network defined as hub genes. For visualization, a heat map was generated using the TOMplot() function in WGCNA R package (version 1.68), with dissimilarity topological overlap (1topological overlap), employed for hierarchical clustering. To generate the network plot, the weights of the network were cut off at 0.1 . Hypergeometric enrichment tests for each module-defined gene list were performed with the enricher() function, as part of clusterProfiler R package version 3.13 .0 ( $\mathrm{Yu}$ et al., 2012). Bonferroni adjustment for $P$-value was used, only gene sets with an adjusted $P$-value $<0.05$ were considered for interpretation of the biological function modules.

\section{Gene set enrichment analysis}

To use the Molecular signatures database (MSigDB), we accessed all gene sets (.gmt file, version 6.2) from the Broad Institute, MA, USA, and chose a subset for further analyses including BioCarta, Hallmark, Gene Ontology (GO), Kyoto Encyclopedia of Genes and Genomes (KEGG), Pathway Interaction Database and the Reactome Pathway Database. To enable cross species comparisons, mouse gene ensembl IDs were converted to human orthologous gene symbols using biomaRt $\mathrm{R}$ package, version 2.41.7 (Durinck et al., 2009). Humanized gene lists for the whole transcriptome were ranked based on logfc values by comparing TdTomato $^{+}$and TdTomato $^{-}$cells. The R package clusterProfiler version 3.13.0 (Yu et al.,
2012) was used to scan through all the gene sets mentioned above, using Benjamini-Hochberg adjusted $P$-values.

\section{Analysis of publicly available scRNA-seq dataset}

scRNA-seq raw expression data was accessed for human mid-gestational brain (22 and 23 weeks post-conception) cortex samples from GSE103723 (Fan et al., 2018). The cells from two individual samples were grouped together, with the sum of reads calculated for each gene to represent the data structure of bulky transcriptomics. The limma package (Ritchie et al., 2015) was used to remove batch effects with removeBatchEffect() and to generate multidimensional scaling plots. To understand the expression pattern of human GREMI and its possible contribution to neural differentiation, normalized counts and tSNE coordinates were employed for visualization using plots generated with ggplot2 package in R environment (Wickham, 2009). To investigate cell types expressing BMP antagonists, Pearson's chisquared test of independence was performed in the $\mathrm{R}$ environment with chisq.test() function. Testing variables were gene expression categorized into high and low by median expression, and using cell type identifiers from the original scRNA-seq study (Fan et al., 2018). Scaled standardized residuals for each gene were used to plot the heatmap using the ComplexHeatmap package (Gu et al., 2016).

To examine the neural cell populations of the cortex expressing Grem 1 throughout developmental stages with single cell resolution in mice, we reanalyzed an scRNA-seq dataset with R version 4.0.3 (Yuzwa et al., 2017). Data were obtained from the Gene Expression Omnibus (GSE107122). We combined E11.5, E13.5, E15.5 and E17.5 together and analyzed with Seurat version 3.0 (Stuart et al., 2019). Data were firstly clustered by setting the dimension 1 to 16 and the resolution at 2 to obtain larger cell numbers within each cluster. Markers for each cluster were calculated through the FindAllMarkers() function, using receiver operating characteristic (ROC) analysis with minimum fraction set at 0.25 and $\log$ fold change set at 0.25 . The top markers for each clusters were compared with the markers list from the original publication and clusters condensed to eight clusters according to the authors' original markers: apical precursor - Sox2, Pax6, Hes1, Hes5, Pcna, Ung; proliferative RGC - Sox2, Pax6, Hes1, Hes5, Slc1a3, Ki67; nonproliferative RGC - Sox2, Pax6, Hes1, Hes5, Slc1a3; excitatory neuron - Tbr1, Tuj1, Satb2, Bhlhe22; newborn neuron - Tbr2, Tuj1, Tbr1, Foxp2, Rein; intermediate progenitor (IP)/excitatory neuron Tbr2, Sstr2, Mfap4, Unc5d, Sema3c, Tuj1, Tbr1; apical IP - Tbr2, Gadd45g, Ngn1 (Neurog1), Ngn2 (Neurog2), Btg2, Sstr2, Mfap4; basal IP Tbr2, Neurod1, Pam, Slc17a6, Sstr2, Mfap4 (Yuzwa et al., 2017). To examine the association between expression of genes of interest (BMP pathway and additional marker genes) and cell clusters, we categorized the expression of genes of interest into high and low expression groups at median expression. We then performed chi-squared tests using base function chisq.test() to look for correlations between gene expression and cell cluster. The standardized residuals for each gene were then visualized using a heatmap with ComplexHeatmap package (Gu et al., 2016).

\section{In situ hybridization}

ISH analyses were performed on frozen mouse tissue samples using RNAscope technology (RNAscope 2.5 HD Detection Kit, Advanced Cell Diagnostics) following the manufacturer's instructions. Briefly, tissue sections were fixed by $4 \%$ paraformaldehyde (PFA) for $15 \mathrm{~min}$, followed by incubation with an $\mathrm{H}_{2} \mathrm{O}_{2}$ solution (Pretreat 1 buffer) for $10 \mathrm{~min}$ at room temperature. Slides were boiled in a target retrieval solution (Pretreat 2 buffer) for $5 \mathrm{~min}$, followed by incubation with a protease solution (Pretreat 3 buffer) for $30 \mathrm{~min}$ at $40^{\circ} \mathrm{C}$. Slides were incubated with a mouse Grem 1 probe and a negative control probe (NM_011824.4, region 398-1359, catalogue number 314741 and 310043 ) for $2 \mathrm{~h}$ at $40^{\circ} \mathrm{C}$, followed by successive incubations with signal amplification reagents. ISH staining was visualized with alkaline phosphatase substrate and Fast Red. Combined ISH/IHC was undertaken by first performing ISH, followed by IHC. For IHC following ISH, the sections were blocked with blocking buffer (X0909, Dako) and then incubated with a primary antibody (rabbit polyclonal antiRFP, Rockland, 600-401-379, 1:1000) overnight at $4^{\circ} \mathrm{C}$. The sections were washed in $1 \times$ phosphate-buffered saline $0.1 \% \mathrm{v} / \mathrm{v}$ Tween (PBST) three times and then incubated with HRP-conjugated secondary antibody (Vector 
Laboratories, PI-1000-1, 1:200) for $60 \mathrm{~min}$ at room temperature. IHC staining was visualized with Universal Elite ABC Kit (PK6101, Vector Laboratories) and DAB (K3468, Dako) and slides were counterstained with hematoxylin, dehydrated in ethanol, cleared in xylene and coverslipped. Cells expressing more than one ISH signal were regarded as positive for Grem1 RNA.

\section{Real time RT-PCR}

RNeasy mini kits (Qiagen) were used to isolate RNA from snap-frozen mouse brain tissues. RNA was reverse-transcribed into cDNA with cDNA master (Sigma-Aldrich). PCR amplification was performed with Kappa Sybr qPCR mix or Kappa Probe qPCR mix using QuantStudio7 (Applied Biosystems). The mouse Gapdh gene was used as an endogenous control. The following primers were used: TaqMan probes and primers - gremlin 1 (IDT, Mm.PT.58.11631114), Id1 (IDT,Mm.PT.58.6622645.g), Id2 (IDT, Mm.PT.58.13116812.g), Id3 (IDT, Mm.PT.58.29482466.g), Id4 (IDT, Mm.PT.58.6851535); Sybr primers - Gapdh-forward AAGGTCATCCCAGAGCTGAA, Gapdh-reverse CTGCTTCACCACCTTCTTGA, Ryr3forward TGCTGTCGCTTCCTTTGCTA, Ryr3-reverse CATCGATGGGGACGCTAGAC, Lrrtm3-forward TAGCAAATCAGGCTCCAGGG, Lrrtm3-reverse GAGTTCATGATGGACCCCACA, $M A G$-forward CCAGTACACCTTCTCGGAGC, $M A G$-reverse TCCGGCACCATACAACTGAC, Gfap-forward ACCGCATCACCATTCCTGTA, Gfap-reverse TGTGACTTTTTGGCCTTCCC, Slc17a7-forward CTTTTTGCGCAGTCGTCACA, Slc17a7-reverse GAGTATCCGACCACCAGCAG.

\section{Immunohistochemistry}

To collect $14.5 \mathrm{dpc}, 17.5 \mathrm{dpc}$ and $20.5 \mathrm{dpc}$ embryonic brains, euthanized pregnant dams immediately underwent cardiac perfusion with $4 \%$ PFA. Whole embryos at $14.5 \mathrm{dpc}$ or dissected embryonic brains for 17.5 and $20.5 \mathrm{dpc}$ were fixed in $4 \% \mathrm{PFA}$ at $4^{\circ} \mathrm{C}$ overnight. Embryos and brains were then cryoprotected in $30 \%$ sucrose and frozen in OCT embedding medium. Then, $16 \mu \mathrm{m}$ sections were cut using a Leica CM1900 cryostat. To make paraffin sections, samples were post fixed in $10 \%$ neutral buffered formalin overnight and processed. Then, $5 \mu \mathrm{m}$ sections were cut using a Leica HM325 microtome. Sections were blocked with Protein Block Serum-Free (Dako) for $1 \mathrm{~h}$ at room temperature, incubated overnight with first antibody at $4^{\circ} \mathrm{C}$, secondary antibody for $1 \mathrm{~h}$ at room temperature, and coverslipped with Vectashield Antifade Mounting Medium (Vector Laboratories). The following antibodies were used: anti-TBR1(Abcam, ab183032, 1:400), antiNeuN (Abcam, ab104225, 1:500), anti- $\beta$ tubulin III (Sigma-Aldrich, T5076, 1:400), anti-O4 (R\&D Systems, MAB1326, 1:500), anti-GFAP (Dako, Z0334, 1:250), anti-FOXP2 (Abcam, ab16046, 1:10,000), antiCtip2 (Abcam, ab18465, 1:800), anti-CDP (Santa Cruz Biotechnology, sc13024, 1:400), anti-BrdU (Abcam, ab6326, 1:600), anti-RFP (Rockland, 600-401-379, 1:1000), anti-Sox2 (Millipore, AB5603, 1:400), anti-Pax6 (Millipore, AB2237, 1:100), anti-TBR2 (Abcam, ab183991, 1:400), antiKi67 (Cell Signaling Technology, \#12202, 1:200), anti-cleaved caspase 3 (Cell Signaling Technology, \#9661, 1:400), anti-phospho SMAD1/5/8 (Millipore, AB3848-I, 1:200). Images were acquired on a Leica SP5 spectral scanning confocal microscope.

\section{Neural stem cell and progenitor cell culture}

Before seeding cells, tissue culture dishes were coated with poly-D-lysine (PDL) $(100 \mu \mathrm{g} / \mathrm{ml})$ and laminin $(10 \mu \mathrm{g} / \mathrm{ml})$. Embryonic NSPCs were isolated from pregnant mice at $14.5 \mathrm{dpc}$. Dorsal telencephalons were dissected from each embryo in PBS, meningeal membranes removed and the tissue triturated to a single cell suspension. Cells were cultured in NeuroCult Proliferation Medium containing $20 \mathrm{ng} / \mathrm{ml}$ epidermal growth factor (EGF) (Stemcell Technologies). For differentiation assays, cells were seeded onto four-well chamber slides (Thermo Fisher Scientific, \#NUN177399) in Neurocult Differentiation medium (Stemcell Technologies). To induce recombination or overexpression of Grem1, cells collected from Grem $1^{\text {flox }}$ flox embryos were infected with plenti-EF1Cre-2a-sfGFP-2a-puro, plenti-EF1-Grem1-2a-sfGFP-2a-puro or plentiEF1-2a-sfGFP-2a-puro lentivirus, and transduced cells were selected using puromycin for 5 days and used for experiments before passage 5 . Lentivirus plasmids psPAX2 and MD2.G were transfected to 293T cells to generate lentivirus, and viral supernatant was concentrated using Amicon-Ultra 100k spin columns. To assess neurosphere forming ability, cells were seeded in 6well uncoated plates $\left(1.6 \times 10^{5}\right.$ cells per well $)$ and cultured for 5 days. The number of neurospheres sized 50-100 $\mu \mathrm{m}$ and $>100 \mu \mathrm{m}$ was counted. For cell viability assays, NSPCs were seeded in 96-well coated plates $\left(1 \times 10^{4}\right.$ cells per well) and RealTime-Glo ${ }^{\mathrm{TM}}$ MT Cell Viability Assay (Promega) was used with the continuous-read protocol at $0,24,52$ and $72 \mathrm{~h}$. Recombinant human BMP2 (Prospec) was added to the differentiation medium.

\section{Luciferase assay}

BMP pathway activity was measured using the BMP response element luciferase reporter pGL3 BRE luciferase (Addgene plasmid \#45126), internal control $\mathrm{pRL} / \mathrm{TK}$-luciferase reporter and the dual luciferase reporter assay kit (Promega). NSPCs were seeded in coated 24-well plates $\left(1.6 \times 10^{5}\right.$ cells per well) and transfected with the reporter plasmids using xTremeGENE HP DNA transfection reagent (Roche). Cells were collected $48 \mathrm{~h}$ after transfection using Passive lysis buffer (Promega). The pGL3 empty vector was used as a control for BMP-independent changes in reporter activity.

\section{Western blot}

Cell lysates were solubilized with M-PER Mammalian Protein Extraction Reagent (Thermo Scientific) containing complete protease and phosphatase inhibitors. Lysates were separated by SDS-PAGE and transferred to PVDF membranes (Millipore). After blocking with 5\% nonfat skim milk in PBST for $30 \mathrm{~min}$ at room temperature, the membranes were incubated overnight at $4^{\circ} \mathrm{C}$ with anti-gremlin 1 antibody (R\&D Systems, AF956, $1: 1000$ ) or anti- $\beta$-actin (Santa Cruz Biotechnology, sc-47778, 1:1000) in $0.5 \%$ nonfat skim milk in PBST. Membranes were washed with PBST and incubated with alkaline phosphatase-conjugated secondary antibodies (anti-rabbit IgG and anti-mouse IgG, GE Healthcare Life Sciences, NA934, NA931, 1:10,000) for $1 \mathrm{~h}$ at room temperature. Finally, the blot was visualized with Immobilon HRP substrate (Millipore) using a Chemi Doc XRS1 (Bio-Rad).

\section{Behavioral tests}

Mice were submitted to Rotarod, open field, elevated plus maze and Y maze tests at the age of 7-10 weeks. Mice in their home cages were acclimatized to the behavior suite for at least $30 \mathrm{~min}$ before testing. The data was acquired blindly to the genotype.

Rotarod: animals were placed on the rotarod (Panlab/Harvard Apparatus) that linearly increased rotation speed from 4 to $40 \mathrm{rpm}$ during a $120 \mathrm{~s}$ period. An accelerating protocol was employed to eliminate the need for habituation to the rotarod. This procedure was repeated for a total of three trials per mouse, separated by $15 \mathrm{~min}$ inter-trial intervals and the mean latency to fall from the rotarod in seconds was compared between wild-type littermate controls and Grem $1^{c K O}$ mice to assess motor coordination.

Open field test: the open field test was conducted in four identical square arenas $(50 \times 50 \times 50 \mathrm{~cm})$ surrounded by walls. Mice were individually placed in a corner of a clean arena and allowed to explore for $10 \mathrm{~min}$. For the purpose of data collection, the arena was conceptually partitioned into two zones: a virtual center zone of $23 \times 23 \mathrm{~cm}$ and a peripheral zone occupying the remaining area. A lower percentage of time spent in the center zone was used to indicate a higher level of anxiety.

Elevated plus maze test: the elevated plus maze consisted of a central square $(8 \times 8 \mathrm{~cm})$ and four arms $(29 \mathrm{~cm}$ long $\times 8 \mathrm{~cm}$ wide, two open arms with no railing and two closed arms enclosed by a transverse wall $20 \mathrm{~cm}$ in height). A mouse was placed in the center of the central square facing the open arm and allowed to explore the maze apparatus for $10 \mathrm{~min}$. The time spent in any of the open arms was recorded and used as a measure of anxiety.

Y-maze test: the apparatus consisted of three arms with an angle of $120^{\circ}$ between each of the two arms. Each arm was $40 \mathrm{~cm}$ long $\times 8 \mathrm{~cm}$ wide $\times 15 \mathrm{~cm}$ high. Visual cues were placed on the walls of the mazes. The Y-maze test consisted of two trials separated by an inter-trial interval (ITI) to assess spatial recognition memory. The first trial (training) had a 5 min duration 
and allowed the mouse to explore only two arms of the maze, with the third arm (novel arm) being blocked. After $30 \mathrm{~min}$ ITI, the second trial was conducted, during which all three arms were accessible for $5 \mathrm{~min}$. Trials were recorded using a ceiling-mounted camera and analyzed by a video analyzer (Ethovision XT, Noldus) to determine the period that each mouse spent in each arm of the maze.

\section{Statistics}

All statistical analyses were performed using Graphpad prism 8, with methods and values summarized in each figure legend.

\section{Acknowledgements}

We thank Dr Randall Grose (SAHMRI, Australia) for flow cytometry assistance; Dr Mark van der Hoek and the Genomics Core (SAHMRI, Australia) for RNA-seq library preparation and sequencing; Dr Hiroaki Fujiwara (The Institute for Adult Diseases, Asahi Life Foundation, Tokyo, Japan) for reviewing the manuscript; Regeneron and Prof Andrew Zannettino for providing Grem $1^{\text {flox }}$ /flox; Emx1-cre mice.

\section{Competing interests}

The authors declare no competing or financial interests.

\section{Author contributions}

Conceptualization: M.I., N.S., D.L.W., S.L.W.; Methodology: M.I., N.S., M.L.; Software: T.W.; Validation: M.I., N.S., J.Q.N., J.A.W., T.R.M.L., K.A.G.; Formal analysis: M.I., N.S., T.W.; Investigation: M.I., N.S., H.K., R.A.; Resources: D.L.W., S.L.W.; Writing - original draft: M.I., N.S., S.L.W.; Writing - review \& editing: M.I., N.S. P.T., D.L.W., S.L.W.; Visualization: M.I., N.S., S.L.W.; Supervision: A.E., S.K., P.T., D.L.W., S.L.W.; Project administration: L.V.; Funding acquisition: D.L.W., S.L.W.

\section{Funding}

M.I. was supported by Japan Society for the Promotion of Science (JSPS) Overseas Research Fellowships and Overseas study grant of Kanzawa Medical Research Foundation; N.S. by fellowship grants from the Astellas Foundation for Research on Metabolic Disorders and the Uehara Memorial Foundation; D.L.W. by a National Health and Medical Research Council (NHMRC) Career Development Fellowship; S.L.W. by the Cancer Council South Australia Beat Cancer Project on behalf of its donors and the State Government of South Australia through the Department of Health (MCF0418 to S.L.W.); and H.K. by JSPS Overseas Challenge Program for Young Researchers, Takeda Science Foundation Fellowship and the Greaton International PhD Scholarship (South Australian Health and Medical Research Institute). This study was supported by grants from the NHMRC (APP1140236 to S.L.W., APP1099283 to D.L.W., APP1143414 to D.L.W./S.L.W.), the JSPS KAKENHI Grant-in-Aid for Scientific Research (19K17478 to N.S.) and the Kanae Foundation for the Promotion of Medical Science Asia-Oceania Collaborative Research Grants to N.S. Open access funding provided by University of Adelaide. Deposited in PMC for immediate release.

\section{Data availability}

All sequencing data reported in this paper have been deposited in GEO under accession number GSE152952.

\section{References}

Ali, I. H. A. and Brazil, D. P. (2014). Bone morphogenetic proteins and their antagonists: current and emerging clinical uses. Br. J. Pharmacol. 171, 3620-3632. doi:10.1111/bph.12724

Bachiller, D., Klingensmith, J., Kemp, C., Belo, J. A., Anderson, R. M., May, S. R., Mcmahon, J. A., Mcmahon, A. P., Harland, R. M., Rossant, J. et al. (2000). The organizer factors Chordin and Noggin are required for mouse forebrain development. Nature 403, 658-661. doi:10.1038/35001072

Baker, A., Kalmbach, B., Morishima, M., Kim, J., Juavinett, A., Li, N. and Dembrow, N. (2018). Specialized subpopulations of deep-layer pyramidal neurons in the neocortex: bridging cellular properties to functional consequences. J. Neurosci. 38, 5441-5455. doi:10.1523/JNEUROSCI.0150-18 2018

Balschun, D., Wolfer, D. P., Bertocchini, F., Barone, V., Conti, A., Zuschratter, W., Missiaen, L., Lipp, H. P., Frey, U. J. and Sorrentino, V. (1999). Deletion of the ryanodine receptor type 3 (RyR3) impairs forms of synaptic plasticity and spatial learning. EMBO J. 18, 5264-5273. doi:10.1093/emboj/18.19.5264

Chen, B., Schaevitz, L. R. and Mcconnell, S. K. (2005). Fezl regulates the differentiation and axon targeting of layer 5 subcortical projection neurons in cerebral cortex. Proc. Natl. Acad. Sci. U.S.A. 102, 17184-17189. doi:10.1073/ pnas.0508732102

Chen, R., Davis, L. K., Guter, S., Wei, Q., Jacob, S., Potter, M. H., Cox, N. J., Cook, E. H., Sutcliffe, J. S. and Li, B. (2017). Leveraging blood serotonin as an endophenotype to identify de novo and rare variants involved in autism. Mol Autism 8, 14. doi:10.1186/s13229-017-0130-3

Choe, Y. and Pleasure, S. J. (2018). Meningeal Bmps regulate cortical layer formation. Brain Plasticity 4, 169-183. doi:10.3233/BPL-170048

Choe, Y., Siegenthaler, J. A. and Pleasure, S. J. (2012). A cascade of morphogenic signaling initiated by the meninges controls Corpus Callosum Formation. Neuron 73, 698-712. doi:10.1016/j.neuron.2011.11.036

Dani, V. S., Chang, Q., Maffei, A., Turrigiano, G. G., Jaenisch, R. and Nelson, S. B. (2005). Reduced cortical activity due to a shift in the balance between excitation and inhibition in a mouse model of Rett syndrome. Proc. Natl. Acad. Sci. USA 102, 12560-12565. doi:10.1073/pnas.0506071102

Durinck, S., Spellman, P. T., Birney, E. and Huber, W. (2009). Mapping identifiers for the integration of genomic datasets with the R/Bioconductor package biomaRt. Nat. Protoc. 4, 1184-1191. doi:10.1038/nprot.2009.97

Fan, X., Dong, J., Zhong, S., Wei, Y., Wu, Q., Yan, L., Yong, J., Sun, L., Wang, X., Zhao, Y. et al. (2018). Spatial transcriptomic survey of human embryonic cerebral cortex by single-cell RNA-seq analysis. Cell Res. 28, 730-745. doi:10.1038/ s41422-018-0053-3

Franco, S. J., Gil-Sanz, C., Martinez-Garay, I., Espinosa, A., Harkins-Perry, S. R., Ramos, C. and Muller, U. (2012). Fate-restricted neural progenitors in the mammalian cerebral cortex. Science 337, 746-749. doi:10.1126/science. 1223616

Fu, C., Li, D., Zhang, X., Liu, N., Chi, G. and Jin, X. (2018). LncRNA PVT1 facilitates tumorigenesis and progression of glioma via regulation of MiR-128-3p/ GREM1 axis and BMP signaling pathway. Neurotherapeutics 15, 1139-1157. doi:10.1007/s13311-018-0649-9

Furuta, Y., Piston, D. W. and Hogan, B. L. (1997). Bone morphogenetic proteins (BMPs) as regulators of dorsal forebrain development. Development 124 2203-2212. doi:10.1242/dev.124.11.2203

Futagi, D. and Kitano, K. (2015). Ryanodine-receptor-driven intracellular calcium dynamics underlying spatial association of synaptic plasticity. J. Comput. Neurosci. 39, 329-347. doi:10.1007/s10827-015-0579-z

Gazzerro, E., Smerdel-Ramoya, A., Zanotti, S., Stadmeyer, L., Durant, D. Economides, A. N. and Canalis, E. (2007). Conditional deletion of gremlin causes a transient increase in bone formation and bone mass. J. Biol. Chem. 282 31549-31557. doi:10.1074/jbc.M701317200

Gomes, W. A., Mehler, M. F. and Kessler, J. A. (2003). Transgenic overexpression of BMP4 increases astroglial and decreases oligodendroglial lineage commitment. Dev. Biol. 255, 164-177. doi:10.1016/S0012-1606(02)00037-4

Gu, Z., Eils, R. and Schlesner, M. (2016). Complex heatmaps reveal patterns and correlations in multidimensional genomic data. Bioinformatics 32, 2847-2849. doi:10.1093/bioinformatics/btw313

Guan, Y., Cheng, W., Zou, C., Wang, T. and Cao, Z. (2017). Gremlin1 promotes carcinogenesis of glioma in vitro. Clin. Exp. Pharmacol. Physiol. 44, 244-256. doi:10.1111/1440-1681.12697

Ha, S., Lee, D., Cho, Y. S., Chung, C., Yoo, Y.-E., Kim, J., Lee, J., Kim, W., Kim, H., Bae, Y. C. et al. (2016). Cerebellar Shank2 regulates excitatory synapse density, motor coordination, and specific repetitive and anxiety-like behaviors. J. Neurosci. 36, 12129-12143. doi:10.1523/JNEUROSCI.1849-16.2016

Haubensak, W., Attardo, A., Denk, W. and Huttner, W. B. (2004). Neurons arise in the basal neuroepithelium of the early mammalian telencephalon: a major site of neurogenesis. Proc. Natl. Acad. Sci. USA 101, 3196-3201. doi:10.1073/pnas. 0308600100

Hevner, R. F., Shi, L., Justice, N., Hsueh, Y.-P., Sheng, M., Smiga, S., Bulfone, A., Goffinet, A. M., Campagnoni, A. T. and Rubenstein, J. L. R. (2001). Tbr regulates differentiation of the Preplate and Layer 6. Neuron 29, 353-366. doi:10. 1016/S0896-6273(01)00211-2

Hsu, D. R., Economides, A. N., Wang, X., Eimon, P. M. and Harland, R. M. (1998) The Xenopus dorsalizing factor Gremlin identifies a novel family of secreted proteins that antagonize BMP activities. Mol. Cell 1, 673-683. doi:10.1016/S10972765(00)80067-2

Imura, T., Tane, K., Toyoda, N. and Fushiki, S. (2008). Endothelial cell-derived bone morphogenetic proteins regulate glial differentiation of cortical progenitors. Eur. J. Neurosci. 27, 1596-1606. doi:10.1111/j.1460-9568.2008.06134.x

Khokha, M. K., Hsu, D., Brunet, L. J., Dionne, M. S. and Harland, R. M. (2003) Gremlin is the BMP antagonist required for maintenance of Shh and Fgf signals during limb patterning. Nat. Genet. 34, 303-307. doi:10.1038/ng1178

Koli, K., Sutinen, E., Rónty, M., Rantakari, P., Fortino, V., Pulkkinen, V., Greco, D., Sipil, P. and Myllárniemi, M. (2016). Gremlin-1 Overexpression in mouse lung reduces silica-induced lymphocyte recruitment - a link to idiopathic pulmonary fibrosis through negative correlation with CXCL10 chemokine. PLOS ONE 11, e0159010. doi:10.1371/journal.pone.0159010

Kowalczyk, T., Pontious, A., Englund, C., Daza, R. A. M., Bedogni, F., Hodge, R., Attardo, A., Bell, C., Huttner, W. B. and Hevner, R. F. (2009). Intermediate neuronal progenitors (basal progenitors) produce pyramidal-projection neurons for all layers of cerebral cortex. Cereb. Cortex 19, 2439-2450. doi:10.1093/cercor/ bhn260

Lamb, T. M., Knecht, A. K., Smith, W. C., Stachel, S. E., Economides, A. N., Stahl, N., Yancopolous, G. D. and Harland, R. M. (1993). Neural induction by 
the secreted polypeptide noggin. Science (New York N.Y.) 262, 713-718. doi:10. $1126 /$ science.8235591

Langfelder, P. and Horvath, S. (2008). WGCNA: an R package for weighted correlation network analysis. BMC Bioinformatics 9, 559. doi:10.1186/1471-2105 9-559

Lee-Hoeflich, S. T., Causing, C. G., Podkowa, M., Zhao, X., Wrana, J. L. and Attisano, L. (2004). Activation of LIMK1 by binding to the BMP receptor, Bmprii, regulates BMP-dependent dendritogenesis. EMBO J. 23, 4792-4801. doi:10. 1038/sj.emboj.7600418

Li, N., Chen, T.-W., Guo, Z. V., Gerfen, C. R. and Svoboda, K. (2015). A motor cortex circuit for motor planning and movement. Nature 519, 51-56. doi:10.1038/ nature 14178

Lim, D. A., Tramontin, A. D., Trevejo, J. M., Herrera, D. G., García-Verdugo, J. M. and Alvarez-Buylla, A. (2000). Noggin antagonizes BMP signaling to create niche for adult neurogenesis. Neuron 28, 713-726. doi:10.1016/S08966273(00)00148-3

Linhoff, M. W., Laurén, J., Cassidy, R. M., Dobie, F. A., Takahashi, H., Nygaard, H. B., Airaksinen, M. S., Strittmatter, S. M. and Craig, A. M. (2009). An unbiased expression screen for Synaptogenic proteins identifies the LRRTM protein family as synaptic organizers. Neuron 61, 734-749. doi:10.1016/j.neuron. 2009.01.017

Marin-Padilla, M. (1978). Dual origin of the mammalian neocortex and evolution of the cortical plate. Anat. Embryol. 152, 109-126. doi:10.1007/BF00315920

Mathieu, C., Sii-Felice, K., Fouchet, P., Etienne, O., Haton, C., Mabondzo, A. Boussin, F. D. and Mouthon, M.-A. (2008). Endothelial cell-derived bone mo rphogenetic proteins control proliferation of neural stem/progenitor cells. Mol. Cell. Neurosci. 38, 569-577. doi:10.1016/j.mcn.2008.05.005

Matsuura, I., Endo, M., Hata, K., Kubo, T., Yamaguchi, A., Saeki, N. and Yamashita, T. (2007). BMP inhibits neurite growth by a mechanism dependent on LIM-kinase. Biochem. Biophys. Res. Commun. 360, 868-873. doi:10.1016/j.bbrc. 2007.06.157

Mikawa, S. and Sato, K. (2011). Noggin expression in the adult rat brain Neuroscience 184, 38-53. doi:10.1016/j.neuroscience.2011.03.036

Mori, H., Ninomiya, K., Kino-Oka, M., Shofuda, T., Islam, M. O., Yamasaki, M., Okano, H., Taya, M. and Kanemura, Y. (2006). Effect of neurosphere size on the growth rate of human neural stem/progenitor cells. J. Neurosci. Res. 84, 1682-1691. doi:10.1002/jnr.21082

Nichols, A. J. and Olson, E. C. (2010). Reelin promotes neuronal orientation and dendritogenesis during preplate splitting. Cereb. Cortex 20, 2213-2223. doi:10. 1093/cercor/bhp303

Reynolds, B. A. and Rietze, R. L. (2005). Neural stem cells and neurospheres-reevaluating the relationship. Nat. Methods 2, 333-336. doi:10.1038/nmeth758

Ritchie, M. E., Phipson, B., Wu, D., Hu, Y., Law, C. W., Shi, W. and Smyth, G. K. (2015). limma powers differential expression analyses for RNA-sequencing and microarray studies. Nucleic Acids Res. 43, e47. doi:10.1093/nar/gkv007

Robinson, M. D., Mccarthy, D. J. and Smyth, G. K. (2010). edgeR: a Bioconductor package for differential expression analysis of digital gene expression data. Bioinformatics 26, 139-140. doi:10.1093/bioinformatics/btp616

Roppongi, R. T., Karimi, B. and Siddiqui, T. J. (2017). Role of LRRTMs in synapse development and plasticity. Neurosci. Res. 116, 18-28. doi:10.1016/j.neures. 2016.10.003

Saiki, A., Sakai, Y., Fukabori, R., Soma, S., Yoshida, J., Kawabata, M., Yawo, H., Kobayashi, K., Kimura, M. and Isomura, Y. (2017). In Vivo Spiking dynamics of intra- and extratelencephalic projection neurons in rat motor cortex. Cereb. Cortex 28, 1024-1038. doi:10.1093/cercor/bhx012

Saxena, M., Agnihotri, N. and Sen, J. (2018). Perturbation of canonical and noncanonical BMP signaling affects migration, polarity and dendritogenesis of mouse cortical neurons. Development 145, dev147157. doi:10.1242/dev.147157

Shen, W., Finnegan, S., Lein, P., Sullivan, S., Slaughter, M. and Higgins, D. (2004). Bone morphogenetic proteins regulate ionotropic glutamate receptors in human retina. Eur. J. Neurosci. 20, 2031-2037. doi:10.1111/j.1460-9568.2004. 03681.x

Söderström, S., Bengtsson, H. and Ebendal, T. (1996). Expression of serine/ threonine kinase receptors including the bone morphogenetic factor type I receptor in the developing and adult rat brain. Cell Tissue Res. 286, 269-279. doi:10.1007/s004410050697

Shipp, S. (2007). Structure and function of the cerebral cortex. Curr. Biol. 17 R443-R449. doi:10.1016/j.cub.2007.03.044

Stuart, T., Butler, A., Hoffman, P., Hafemeister, C., Papalexi, E., Mauck, W. M., Hao, Y., Stoeckius, M., Smibert, P. and Satija, R. (2019). Comprehensive integration of single-cell data. Cell 177, 1888-1902.e21. doi:10.1016/j.cell.2019. 05.031

Sun, Y. and Xu, Z. (2010). Regulation of neural stem cell by bone morphogenetic protein (BMP) signaling during brain development. Front. Biol. 5, 380-385. doi:10. 1007/s11515-010-0860-5

Sun, M., Thomas, M. J., Herder, R., Bofenkamp, M. L., Selleck, S. B. and O'connor, M. B. (2007). Presynaptic contributions of chordin to hippocampal plasticity and spatial learning. J. Neurosci. 27, 7740-7750. doi:10.1523/ JNEUROSCI.1604-07.2007

Um, J. W., Choi, T.-Y., Kang, H., Cho, Y. S., Choii, G., Uvarov, P., Park, D., Jeong D., Jeon, S., Lee, D. et al. (2016). LRRTM3 regulates excitatory synapse development through alternative splicing and neurexin binding. Cell Rep. 14 808-822. doi:10.1016/j.celrep.2015.12.081

Wang, W., Andolina, I. M., Lu, Y., Jones, H. E. and Sillito, A. M. (2016). Focal gain control of thalamic visual receptive fields by layer 6 corticothalamic feedback. Cereb. Cortex 28, 267-280. doi:10.1093/cercor/bhw376

Wickham, H. (2009). Ggplot2: Elegant Graphics for Data Analysis. New York: Springer.

Wilson, S. W. and Houart, C. (2004). Early Steps in the Development of the Forebrain. Dev. Cell 6, 167-181. doi:10.1016/S1534-5807(04)00027-9

Wood, L. and Shepherd, G. M. G. (2010). Synaptic circuit abnormalities of motorfrontal layer $2 / 3$ pyramidal neurons in a mutant mouse model of Rett syndrome. Neurobiol. Dis. 38, 281-287. doi:10.1016/j.nbd.2010.01.018

Worthley, D. L., Churchill, M., Compton, J. T., Tailor, Y., Rao, M., Si, Y., Levin, D. Schwartz, M. G., Uygur, A., Hayakawa, Y. et al. (2015). Gremlin 1 identifies a skeletal stem cell with bone, cartilage, and reticular stromal potential. Cell 160 269-284. doi:10.1016/j.cell.2014.11.042

Xiao, L., Michalski, N., Kronander, E., Gjoni, E., Genoud, C., Knott, G. and Schneggenburger, R. (2013). BMP signaling specifies the development of a large and fast CNS synapse. Nat. Neurosci. 16, 856-864. doi:10.1038/nn.3414

Yamasaki, A., Kasai, A., Toi, A., Kurita, M., Kimoto, S., Hayata-Takano, A., Nakazawa, T., Nagayasu, K., Shintani, N., Hashimoto, R. et al. (2015). Identification of the role of bone morphogenetic protein (BMP) and transforming growth factor- $\beta$ (TGF- $\beta$ ) signaling in the trajectory of serotonergic differentiation in a rapid assay in mouse embryonic stem cells in vitro. J. Neurochem. 132, 418-428. doi:10.1111/jnc.12999

Yan, K., Wu, Q., Yan, D. H., Lee, C. H., Rahim, N., Tritschler, I., Devecchio, J. Kalady, M. F., Hjelmeland, A. B. and Rich, J. N. (2014). Glioma cancer stem cells secrete Gremlin1 to promote their maintenance within the tumor hierarchy Genes Dev. 28, 1085-1100. doi:10.1101/gad.235515.113

Yu, G., Wang, L.-G., Han, Y. and He, Q.-Y. (2012). clusterProfiler: an R package for comparing biological themes among gene clusters. OMICS: A J. Integr. Biol. 16, 284-287. doi:10.1089/omi.2011.0118

Yun, K., Mantani, A., Garel, S., Rubenstein, J. and Israel, M. A. (2004). Id4 regulates neural progenitor proliferation and differentiation in vivo. Development 131, 5441-5448. doi:10.1242/dev.01430

Yuzwa, S. A., Borrett, M. J., Innes, B. T., Voronova, A., Ketela, T., Kaplan, D. R. Bader, G. D. and Miller, F. D. (2017). Developmental emergence of adult neura stem cells as revealed by single-cell transcriptional profiling. Cell Rep. 21 3970-3986. doi:10.1016/j.celrep.2017.12.017 